\title{
Geosynthetic capillary barriers: current state of knowledge
}

\author{
J. G. Zornberg ${ }^{1}$, A. Bouazza ${ }^{2}$ and J. S. McCartney ${ }^{3}$ \\ ${ }^{1}$ Fluor Centennial Associate Professor, The University of Texas at Austin, Civil Engineering \\ Department-GEO, 1 University Station C1792 Austin, TX 78712-0280, USA, Telephone: +1 512232 \\ 3595, Telefax: +1 512471 6548,E-mail: zornberg@mail.utexas.edu \\ ${ }^{2}$ Associate Professor, Monash University, Melbourne, Australia, Telephone: +61 39905 4956, Telefax: \\ +61 39905 4944, E-mail: malek.bouazza@eng.monash.edu.au \\ ${ }^{3}$ Assistant Professor and Barry Faculty Fellow, University of Colorado at Boulder, Department of Civil, \\ Environmental, and Architectural Engineering, UCB 428, Boulder, CO 80309, USA, Telephone: +1 303 \\ 492 0492, Telefax: +1 303492 7317,E-mail: john.mccartney@colorado.edu
}

Received 5 January 2010, revised 30 July 2010, accepted 30 July 2010

\begin{abstract}
This paper provides theoretical background, laboratory data and full-scale measurements useful in understanding the interaction between soils and geosynthetics under unsaturated conditions. It also includes an evaluation of the current state of knowledge regarding the hydraulic properties of porous geosynthetics under unsaturated conditions relevant for geosynthetic capillary barrier design. These properties include the water retention curve and the hydraulic conductivity function. In addition, the mechanisms involved in the development of capillary barriers are evaluated to explain the storage of water at the interface between materials with contrasting hydraulic conductivity (e.g. a fine-grained soil and a nonwoven geotextile). Finally, specific applications are presented to illustrate new opportunities and applications that may result from a better understanding of the unsaturated hydraulic properties of geosynthetics. Experimental data are provided illustrating that geosynthetic capillary barriers are superior to soil-only capillary barriers. Based on this observation, it is emphasized that no capillary barrier should be designed without consideration of the enhanced performance offered by the inclusion of nonwoven geotextiles under the fine-grained soil component of the cover.
\end{abstract}

KEYWORDS: Geosynthetics, Geotextiles, Unsaturated soil, Water retention curve, Hydraulic conductivity, Capillary barriers

REFERENCE: Zornberg, J. G., Bouazza, A. \& McCartney, J. S. (2010). Geosynthetic capillary barriers: current state of knowledge. Geosynthetics International, 17, No. 5, 273-300.

[doi: 10.1680/gein.2010.17.5.273]

\section{INTRODUCTION}

Capillary barriers are becoming an established technology to control water flow in unsaturated soils. A capillary barrier develops when an unsaturated fine-grained soil layer is underlain by another unsaturated porous material with relatively large-sized pores, such as a coarse-grained soil layer (e.g. sand or gravel), or a porous geosynthetic (e.g. a nonwoven geotextile). This phenomenon was first observed by Kisch (1959) and subsequently in layered soil profiles in geotechnical applications (Rasmusson and Eriksson 1987; Nicholson et al. 1989; Barbour 1990; Shackelford et al. 1994; Yanful 1993; Woyshner and Yanful 1995). The capillary break effect that develops in systems involving geosynthetics (e.g. geosynthetic drainage layers, GDLs) has been evaluated only more recently.
The main impact of the capillary break effect on an unsaturated soil-GDL system is that a measurable amount of water will not flow from the soil into the underlying GDL until a critical condition is reached (Stormont 1995; Bouazza et al. 2006a). Because of this, the capillary break effect has been observed to increase the water storage capacity of soils beyond the level that would normally drain under gravity (Stormont and Morris 1998; Khire et al. 2000).

Key to the understanding of capillary barriers is the assessment of water flow and storage in porous geomaterials (e.g. soils, geosynthetics) under unsaturated conditions. As a geomaterial desaturates, its hydraulic conductivity does not remain constant but, instead, drops significantly with decreasing degrees of saturation. The hydraulic conductivity of unsaturated geomaterials with 
relatively large pores (e.g. gravel, geotextiles) decreases faster than that of fine-grained soils. This characteristic leads to the counterintuitive situation in which the hydraulic conductivity of unsaturated gravel or geotextiles can be significantly smaller than that of fine-grained soils (e.g. orders of magnitude smaller than the saturated hydraulic conductivity of the bentonite component of GCLs).

Understanding of the concepts of water flow in unsaturated soils has gained added relevance in landfill design because of the increased number of alternative covers that have been recently designed and constructed for waste containment and mine-tailing facilities. Specifically, capillary barriers have recently been used in lieu of geomembrane liners in multiple projects located in arid and semi-arid sites (e.g. in the western United States, Western Australia, and South Africa). It turns out that not only geosynthetics can be used in capillary barrier systems but, as will be demonstrated in this paper, they provide performance superior to that of soil-only capillary barriers. Geosynthetic capillary barriers have been recently permitted, designed and constructed in high-visibility, hazardous waste facilities in the United States. Although the focus of this paper is on the use of geosynthetic capillary barriers for landfill and mine-tailing covers, it should be recognized that an understanding of water flow in unsaturated geosynthetics can also be useful in the design of optimized drainage layers, pavement systems, reinforcedsoil structures involving poorly draining backfills, and agricultural applications, all of which involve unsaturated soils.

The design of cover systems involving capillary barriers relies heavily on the quantification of atmospheric processes and water flow though unsaturated geomaterials (soil or geosynthetics). In the United States, the design of final cover systems for new municipal and hazardous waste containment systems is prescribed by the U.S. Resource Recovery and Conservation Act (RCRA) Subtitles D and C, respectively. Federal- and state-mandated cover systems for municipal and hazardous waste landfills have endorsed the use of resistive barriers. Resistive cover systems involve a liner (e.g. a compacted clay layer) constructed with a soil of low saturated hydraulic conductivity (typically $10^{-9} \mathrm{~m} / \mathrm{s}$ or less) to reduce basal percolation. Figure 1a shows the water balance components in a resistive system, in which basal percolation control is achieved by maximizing overland runoff. In order to enhance cover performance and lower construction costs, RCRA regulations allow the use of alternative cover systems if comparative analyses and/or field demonstrations can satisfactorily show their equivalence to prescriptive systems. Evapotranspirative covers are alternative systems that have recently been implemented in several high-profile sites in various parts of the world. Evapotranspirative covers are vegetated with native plants that survive on the natural precipitation, and have been shown to be stable over long periods of time. Figure $1 \mathrm{~b}$ illustrates the water balance components in an evapotranspirative cover system. Evapotranspiration and water storage are components that significantly influence the performance of this system. Internal lateral drainage may



(a)

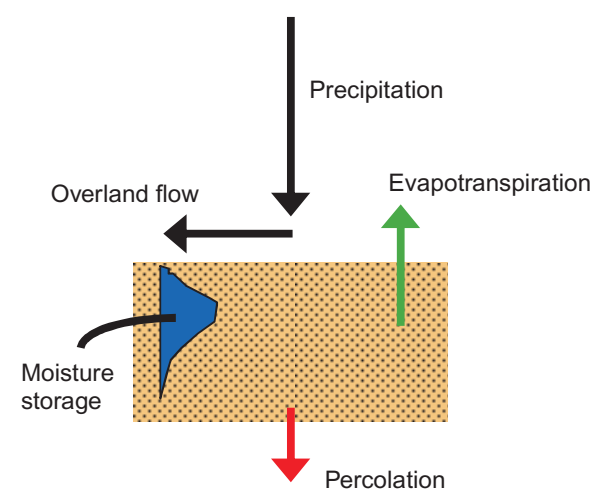

(b)

Figure 1. Components of the water balance in cover systems: (a) resistive barrier; (b) evapotranspirative cover

also be a relevant component in some cover types (capillary barriers on steep slopes). The novelty of this approach is the mechanism by which basal percolation control is achieved: an evapotranspirative cover acts not as a barrier, but as a sponge or a reservoir that stores water during precipitation events, and then releases it back to the atmosphere as evapotranspiration or lateral drainage. Silts and clays of low plasticity are the soils most commonly used in evapotranspirative covers, as they can store water while minimizing the potential for cracking upon desiccation.

Additional advantages of evapotranspirative covers over clay barrier systems include a low potential for desiccation cracking, easy construction, and low maintenance. Also, evapotranspirative covers can be constructed with a reasonably broad range of soils, contributing to cost savings associated with the use of site-specific soils instead of imported material. The performance of evapotranspirative cover systems has been documented by field experimental assessments (Anderson et al. 1993; Dwyer 1998), and procedures have been developed for quantitative evaluation of the variables governing their performance (Khire et al. 2000; Zornberg et al. 2003).

The increased use of alternative cover systems in recent years has led to concerns within the geosynthetics industry, as implementation of these covers can potentially result in decreased use of geosynthetics in landfill cover projects. In particular, the Geosynthetic Research Institute has issued a White Paper on 'the questionable strategy of soil-only landfill covers' (GRI 2003). The White Paper was prepared in response to the reported findings of a 
large-scale field study, funded by the U.S. Department of Energy at Sandia National Laboratories, which compared the performance of composite (CCL-geomembrane) covers with that of capillary barriers, anisotropic and monolithic covers (Dwyer 1998, Dwyer 2001). The heart of the controversy lay in the fact that holes were purposely made in the geomembranes of the composite cover system. Independent of the results of that particular study, an understanding of the capillary break induced by nonwoven geotextiles may place a different perspective on the overall role of geosynthetics in alternative covers. As will be discussed in this paper, this is because: (1) much of the field instrumentation data documenting the good performance of evapotranspirative covers has been based on lysimeters, which significantly underestimate the basal flow due to the development of a capillary break (see Section 6.2.1); and (2) available research has recently shown that the use of nonwoven geotextiles in a capillary barrier system provides superior performance when compared with the use of traditional coarse-grained soils (see Section 6.2.2).

This paper includes an evaluation of the current state of knowledge of the hydraulic properties of geosynthetics under unsaturated conditions that are relevant for geosynthetic capillary barrier design. These properties include the water retention curve and the hydraulic conductivity function. In addition, the mechanisms involved in the development of capillary barriers are evaluated to explain the storage of water that develops at the interface between materials with contrasting hydraulic conductivity (e.g. a fine-grained soil and a nonwoven geotextile). Finally, specific applications and case histories are discussed to illustrate new opportunities that may result from a better understanding of the unsaturated hydraulic properties of geosynthetics.

\section{WATER FLOW THROUGH UNSATURATED GEOMATERIALS}

The principles of water flow through unsaturated geomaterials are more complex than those for water through saturated media. This is partly because the most important variable that governs the rate of water flow through geomaterials (i.e. the hydraulic conductivity) is not constant with varying water storage. Instead, the hydraulic conductivity under unsaturated conditions varies with the level of suction (or water content) within the geomaterial. Consequently, relative amounts of water and air in the geomaterial significantly influence the geomaterial hydraulic behavior. The water content is typically quantified on a volumetric basis in the case of problems involving flow of water in unsaturated soils. The volumetric water content, $\theta$, is defined as the ratio between the volume of water and the total control volume. The porosity, $n$, which is the ratio between the volume of voids and the total control volume, corresponds to the volumetric water content at saturation (i.e. $n=\theta_{\mathrm{s}}$ ). The degree of saturation $S$, commonly used to normalize the water content of a geomaterial is the ratio between the volumetric water content and the porosity.
In the case of unsaturated soils, water is held within the pores against the pull of gravity by a combination of adsorptive and capillary pressures (Olson and Langfelder 1965). Adsorptive pressures are present in soils as a result of electrical fields and short-range attractive forces (van Der Waals forces) that tend to draw water toward the soil particles. These pressures may be significant in highly plastic clays, where the net negative charges on the surface of clay particles interact with water dipoles and cations in the pore water. In the case of granular soils and geosynthetics, only capillary pressures are relevant. The adsorptive and capillary pressures are considered together as a single variable, referred to as the matric suction $\psi$, which has units of pressure $(\mathrm{kPa})$. The matric suction in a geomaterial is quantified as the difference between the pore air pressure and the pore water pressure. Water is a wetting fluid for most soil minerals and geosynthetic polymers, implying that the air/water menisci between individual soil particles or geosynthetic fibers are convex, tensioned membranes (Henry and Patton 1998). Accordingly, the air pressure is greater than the water pressure, so the water pressure has a negative magnitude when air is under atmospheric pressure. The capillary rise in a pipette provides an analogy useful to assess the influence of pore sizes on the matric suction. In this case, the matric suction $\psi$ is given by the expression

$$
\psi=P_{\mathrm{a}}-P_{w}=h_{\mathrm{c}} \rho_{\mathrm{w}} g=\frac{2 \sigma_{\mathrm{aw}} \cos \gamma}{R}
$$

where $P_{\mathrm{a}}$ is the pore air pressure, $P_{\mathrm{w}}$ is the pore water pressure, $h_{\mathrm{c}}$ is the height of capillary rise in a pipette of radius $R, \rho_{\mathrm{w}}$ is the density of water, $g$ is the acceleration of gravity, $\sigma_{\mathrm{aw}}$ is the surface tension between water and air, and $\gamma$ is the wetting contact angle (typically $10^{\circ}$ for quartz minerals). Equation 1 assumes that air is under atmospheric pressure $\left(P_{\mathrm{a}}=0\right)$, and indicates that the suction is inversely proportional to the pore radius $R$ (note that all other variables are constants). Accordingly, for the same volumetric water content, a fine-grained soil (with comparatively small pore radii) will have a higher suction than a coarse-grained soil or a nonwoven geotextile (with comparatively large pore radii). The relationship between water content and suction is thus related to the pore size distribution of the geomaterial. In engineering practice, the relationship between water content and suction is referred to as the water retention curve $\theta(\psi)$ (WRC).

The flow of water through geomaterials is driven by a gradient in the hydraulic energy, which can be quantified by the fluid potential (energy per unit mass of water). The fluid potential is given by an expanded form of Bernoulli's equation,

$$
\Phi=g z+\frac{1}{2}\left(\frac{v}{n}\right)^{2}+\frac{(-\psi)}{\rho_{\mathrm{w}}}+\frac{P_{\mathrm{o}}}{\rho_{\mathrm{w}}}
$$

where $\Phi$ is the fluid potential (i.e. energy per unit mass of fluid), $z$ is the vertical distance from the datum, $v$ is the water discharge velocity, $n$ is the porosity, and $P_{\mathrm{o}}$ is the osmotic suction. In Equation 2 the four terms on the righthand side correspond to the potential energy, the kinetic 
energy, the energy due to the water suction $\left(\psi=-P_{\mathrm{w}}\right.$ if $P_{\mathrm{a}}=0$ ), and the energy due to the osmotic suction. The water seepage velocity $(v / n)$ is comparatively small, so the kinetic energy component is negligible. The osmotic suction is typically considered constant, and consequently does not lead to a contribution to the hydraulic potential gradient. The energy due to water suction is often the governing term. As in the case of water-saturated geomaterials, Darcy's law for unsaturated geomaterials indicates that flow is driven by the gradient in total hydraulic potential. However, the available pathways for water flow in an unsaturated geomaterial decrease as the water content decreases (or as suction increases). This is quantified by the hydraulic conductivity function $K(\psi)$ (the $K$ function), which accounts for the decrease in conductivity with increasing suction (or decreasing water content). The water discharge velocity through a geomaterial in the vertical direction $z$ can be estimated using Darcy's law and Equation 2, as follows:

$$
\begin{aligned}
v & =\frac{Q}{A}=-\frac{K(\psi)}{g} \frac{\partial \Phi}{\partial z} \\
& =-K(\psi) \frac{\partial}{\partial z}\left(1-\frac{1}{\rho_{\mathrm{w}} g} \frac{\partial \psi}{\partial z}\right)
\end{aligned}
$$

where $Q$ is the volumetric flow rate, and $A$ is the area of geomaterial perpendicular to the flow direction. The continuity principle in this control volume can be expressed by

$$
\frac{\partial \theta(\psi)}{\partial t}=-\frac{\partial v}{\partial z}
$$

In Equation 4 the left-hand side represents the change in water storage in the control volume with time, and the right-hand side represents the change in flow rate with distance across the control volume. Substitution of Equation 3 into Equation 4 leads to the governing equation for one-dimensional flow through unsaturated porous materials, referred to as Richards' equation:

$$
\frac{\partial \theta(\psi)}{\partial \psi} \frac{\partial \psi}{\partial t}=\frac{\partial}{\partial z}\left[K(\psi)\left(1-\frac{1}{\rho_{\mathrm{w}} g} \frac{\partial \psi}{\partial z}\right)\right]
$$

Richards' equation is a coupled, nonlinear parabolic equation, which can be solved using finite differences or finite elements. Inspection of Richards' equation illustrates that suction is the primary variable used in the analysis of water flow through unsaturated soils. Numerical solutions to Richards' equation can be challenging, because the constitutive functions $\theta(\psi)$ and $K(\psi)$ are highly nonlinear, and may have undefined or zero derivatives. More importantly, the constitutive functions $\theta(\psi)$ and $K(\psi)$ need to be obtained experimentally. In the case of geosynthetics, recent research efforts have been undertaken to obtain experimentally $\theta(\psi)$ (the WRC: see Section 3.1) and $K(\psi)$ (the $K$-function: see Section 3.2).

As will be discussed, the hydraulic conductivity of nonwoven geotextiles under unsaturated conditions is typically lower than that of most soils, working then as hydraulic barriers. Nonwoven geotextiles are effective as hydraulic barriers for unsaturated soils for the same reason that makes them effective for separation, protection and drainage: their pore size is greater than that of most soils. The specific phenomenon that resists the passage of water from an unsaturated soil into a nonwoven geotextile is referred to as the capillary break effect (Stormont and Anderson 1999). The capillary break effect is encountered in everyday life with baby bottles, as milk will not pour freely from the small hole of an inverted bottle through the relatively large opening of air on the other side of the nipple. Even though the water pressure on the milk side of the nipple equals the hydrostatic pressure of milk in the bottle, an additional suction must be applied on the air side of the nipple to induce flow. The capillary break effect occurs at the interface between fine-grained materials having relatively small pores and coarse-grained materials having relatively large pores, as shown schematically in Figure 2. The radius of the air/water meniscus changes from $r_{1}$ to $r_{2}$ at the interface between the small and large pores. Inspection of Equation 1 indicates that the small soil pores of radius $r_{1}$ result in comparatively large suction, and water can move to the larger geotextile pores only when a significantly smaller suction (corresponding to the larger radius $r_{2}$ of the geotextile) develops in the system. In other words, the energy in the pore water has to be sufficiently high to permit it to break into the large pore. Macroscopically, the capillary break effect prevents a measurable amount of water from flowing from the soil into the nonwoven geotextile until a critical suction close to zero (saturation) is reached. At this point, water is capable of 'breaking' into the large pore from the small pore.

Early field evidence of the impact of the capillary break was reported by Clough and French (1982), who realized that nonwoven geotextiles used as under-drains in unbound pavements led to an increase in the water content
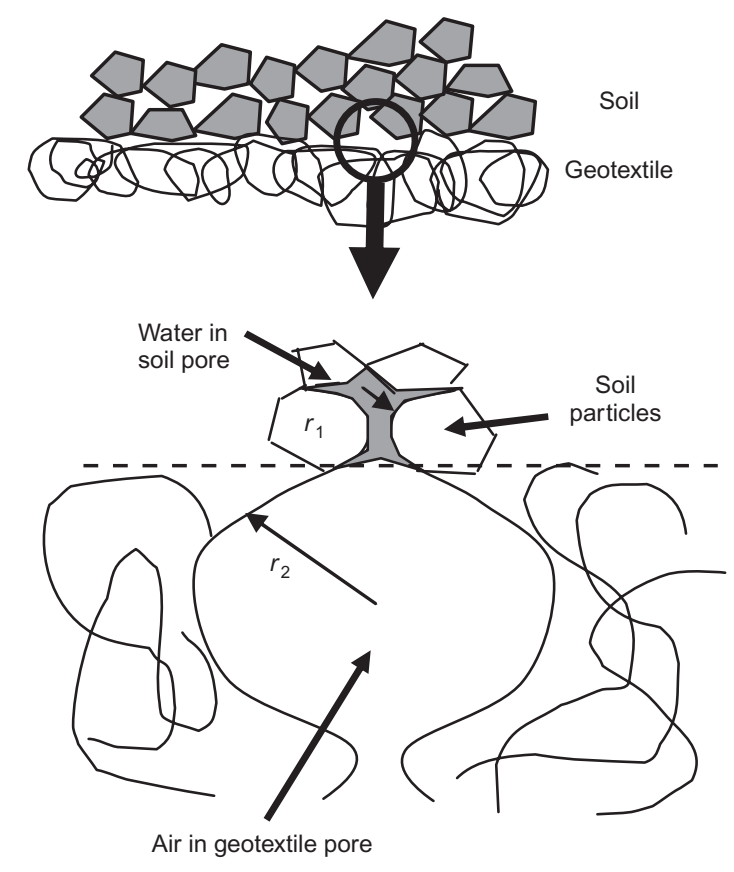

Figure 2. Schematic of the conditions leading to a capillary break effect at the soil/geotextile interface 
of the overlying soils. Similarly, Richardson (1997), in another early observation, reported that nonwoven geotextiles used in a slope drainage layer did not drain water readily from overlying unsaturated soils until the soil was nearly saturated. The failure of the soil-geosynthetic system was attributed to this phenomenon, as the designer had not considered the increased unit weight of the nearly saturated soil on the slope.

\section{HYDRAULIC PROPERTIES OF UNSATURATED GEOTEXTILES}

Among the various types of geosynthetics, geotextiles have been used in geotechnical engineering applications to fulfill the widest range of functions (Koerner 2005; Zornberg and Christopher 2007). This includes separation between different soil layers, protection of geomembranes or other geosynthetics from puncture, drainage from surrounding soil, and reinforcement of poorly draining backfills. Geotextiles are able to meet these requirements despite their small thickness (e.g. $2.5 \mathrm{~mm}$ ), partly as a result of their high porosity (typically about 0.9 ), which is greater than that of most soils. Geotextiles have a uniform pore size compared with most soils (Palmeira and Gardoni 2002; Aydilek et al. 2007). In addition, important hydraulic properties of unsaturated geotextiles include their water retention curve and their hydraulic conductivity function.

\subsection{Water retention curve}

The water storage of soil and geosynthetics is typically quantified using the relationship between volumetric water content and suction, referred to as the water retention curve (WRC). Figure 3 shows the WRCs for different geotechnical materials. The coarser materials (sand and geotextile) show a highly nonlinear response, with a significant decrease in water content (or degree of saturation) within a comparatively narrow range of suction. The fine-grained soil shows instead a more gradual decrease in water content with increasing suction. The nonlinearity observed in these relationships is caused partly by the range of pore size distributions in these materials. During initial drying of a fully saturated geomaterial, the negative pressure in the water increases, but water does not flow

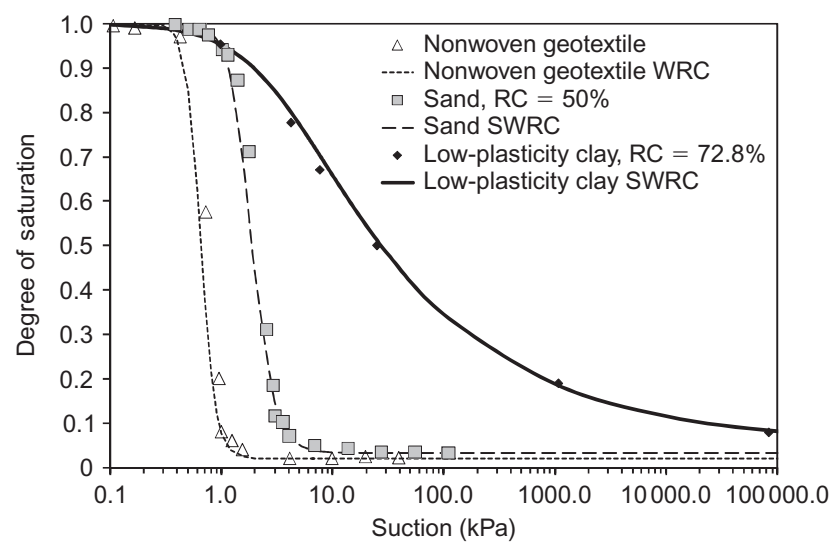

Figure 3. Typical WRCs for different geotechnical materials (after McCartney et al. 2005) from the geomaterial until the value of suction corresponding to the air entry value is reached. When this suction value is reached, air enters the specimen and the water content decreases. After the air entry value is reached, the water content drops from saturation to a value that remains approximately constant with increasing suction. This low water content value is often referred to as the residual water content. The residual condition occurs because the water becomes occluded (or disconnected) within the soil pores, with no available pathways for water to flow.

The WRC for a given material is sensitive not only to the pore size distribution, but also to the soil mineralogy (for soils), polymeric material (for geosynthetics), density, and pore structure (Hillel 1998; Bouazza et al. 2006a, 2006b). The WRC can show significantly different wetting and drying paths, a phenomenon referred to as hysteresis (Topp and Miller 1966; Kool and Parker 1987; Bouazza et al. 2006a). During drying, the largest pores drain first, followed by the smaller pores. During wetting, the smaller pores fill first, but the presence of large pores may prevent some of the small pores from filling. Also, wetting of a dry geomaterial often leads to entrapment of air in the larger pores, preventing saturation of the medium unless positive pressure is applied to the water. Air entrapment causes the wetting path to be relatively flat for high suction, with a steep increase in volumetric water content at lower suctions. Figure 4 shows the WRCs of three geotextiles, illustrating the significant hysteresis in their response to wetting and drying (Bouazza et al. 2006a). Recent experimental results have also highlighted the impact on hysteresis of the direction of flow measurement (Nahlawi 2009). In particular, it was found that the volumetric water content of geotextiles along the crossplane direction differed from that obtained along the in-plane direction for the same suction head. Several techniques have been developed to determine the WRC of soils experimentally (Wang and Benson 2004; Klute 1986). These techniques have recently been adapted to obtain the WRC of geotextiles experimentally. Two main groups of techniques that have been used to define the WRC are physical techniques and thermodynamic techniques.

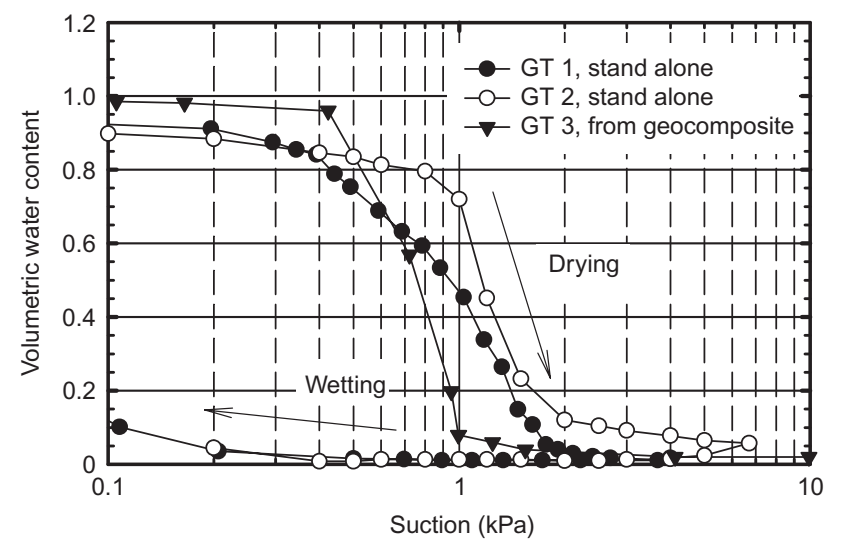

Figure 4. Typical geotextile water retention curves (WRCs) (Bouazza et al. 2006a) 
The first group of techniques (physical techniques) involves an initially water-saturated geomaterial from which water is slowly expelled by imposing suction on a specimen boundary. Flow continues until a condition is reached in which the water content and suction are in equilibrium. The most commonly used physical technique for geotextiles is the hanging column test. A schematic of a typical hanging column used for soils is shown in Figure 5a. This test involves a ceramic plate that is connected to a manometer tube. A negative pressure is imposed on the ceramic plate by holding the water level in the manometer tube beneath the plate. Stormont et al. (1997) applied the experimental technique available for soils to measure the water retention functions of geotextiles. The testing apparatus is similar to the hanging column apparatus used for testing the water retention functions of soils as presented by Klute (1986). The apparatus consists of a Buchner filter funnel fitted with a porous plate, a bottle acting as a water reservoir, and tubing used to connect the bottom of the funnel to the bottom of the bottle, as well as the top of the funnel to the top of the bottle. The original



(a)

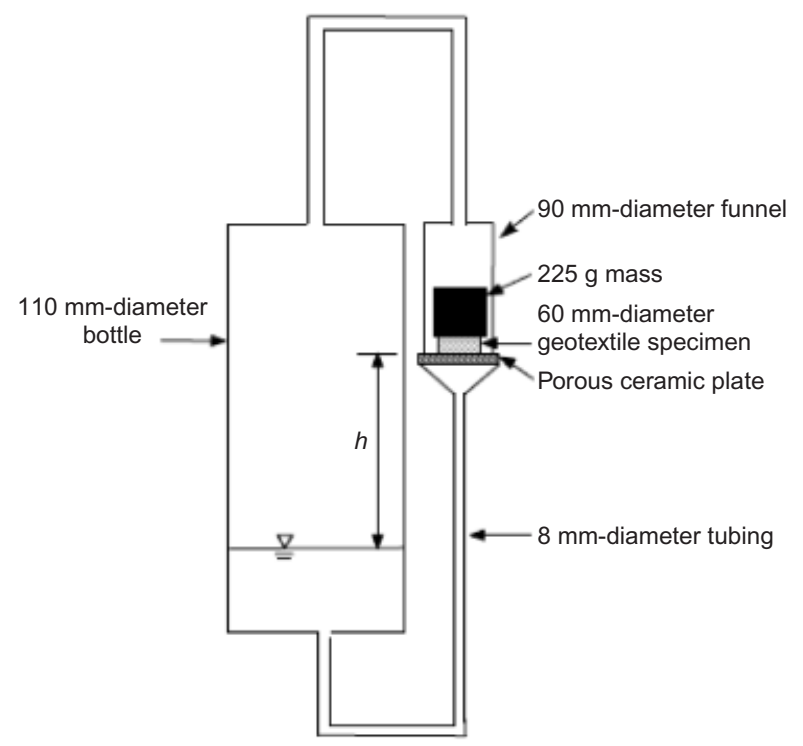

(b)

Figure 5. Hanging column test: (a) conventional test used for soil specimens; (b) modified test used for geotextile specimens (Stormont et al. 1997) test schematic as presented by Stormont et al. (1997) is shown in Figure 5b. The geotextile specimen is placed on the porous ceramic plate under a seating load to facilitate contact between the two porous materials. The porous plate is initially saturated and connected to the reservoir. The funnel is moved to different elevations above the air/ water interface in the reservoir to impose a target suction value on the geotextile specimen. The specimen is removed from the testing apparatus after equilibrating at a desired suction (typically $24 \mathrm{~h}$ ), and weighed to determine the water content at the target suction. The measured values of suction and water content are then used to define one point of the WRC. Other variations of the hanging column test have been reported by McCartney et al. (2005) and Bouazza et al. (2006a, 2006b). Variations include flushing of the geotextile samples with $\mathrm{CO}_{2}$, and extended equilibration times.

Another common physical technique is the axistranslation or pressure plate test. Figure 6a shows the typical setup used for soil testing, which involves placing a soil specimen on a ceramic plate that conducts only water and applying air pressure to the specimen. The air pressure leads to a hydraulic gradient in matric suction in the specimen, causing the pore water to flow through the ceramic plate. At equilibrium, the air pressure corresponds to the capillary pressure, since the water pressure is kept equal to zero. The outflow volume is then measured using a constant-head Mariotte bottle. This approach is repeated for successively higher pressures that gradually dry the specimen. The pressure may be subsequently decreased to measure the wetting behavior. At the end of testing the gravimetric water content is measured destructively, and the water content at each pressure increment can be backcalculated from the outflow measurements. Additional details regarding the testing procedure can be found in the ASTM standard for WRC determination (ASTM D6836). Knight and Kotha (2001) modified the technique used for soils to measure the water retention function of nonwoven geotextile specimens. Nahlawi et al. (2007a) and Bathurst et al. (2009) further modified the capillary pressure cell to accommodate large-diameter samples and to control very accurately the air pressure through a pneumatic pressure controller. Before testing, the geotextile and the porous ceramic disc were flushed with $\mathrm{CO}_{2}$ to facilitate the solution of air bubbles in the wetting phase. The test apparatus is shown schematically in Figure $6 b$.

The second group of techniques (thermodynamic techniques) involves allowing water to evaporate from a specimen in a closed chamber under controlled relative humidity. The relative humidity is controlled by allowing water to evaporate from a saturated salt solution placed within the chamber, as shown in Figure 7. Another commonly used thermodynamic technique is the chilledmirror hygrometer (Wang and Benson 2004). This device infers the total soil suction (matric and osmotic) by measuring the vapor pressure in the soil, which is related to the temperature at which water condenses on a mirror. When condensation occurs, a change in the optical properties of the mirror is detected. In general, physical techniques are used for relatively low suctions (i.e. under 


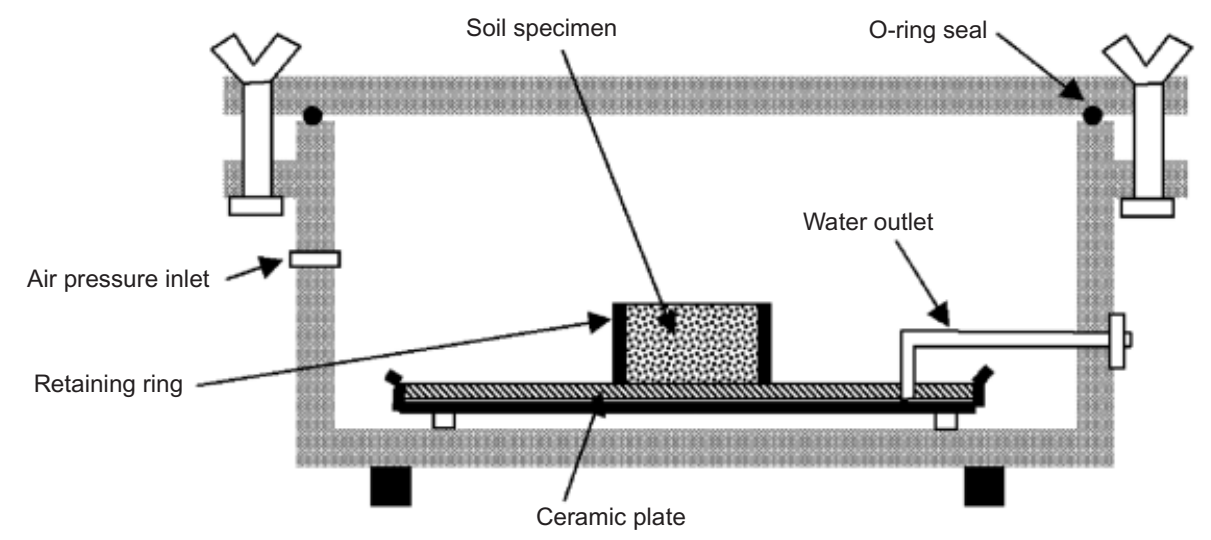

(a)

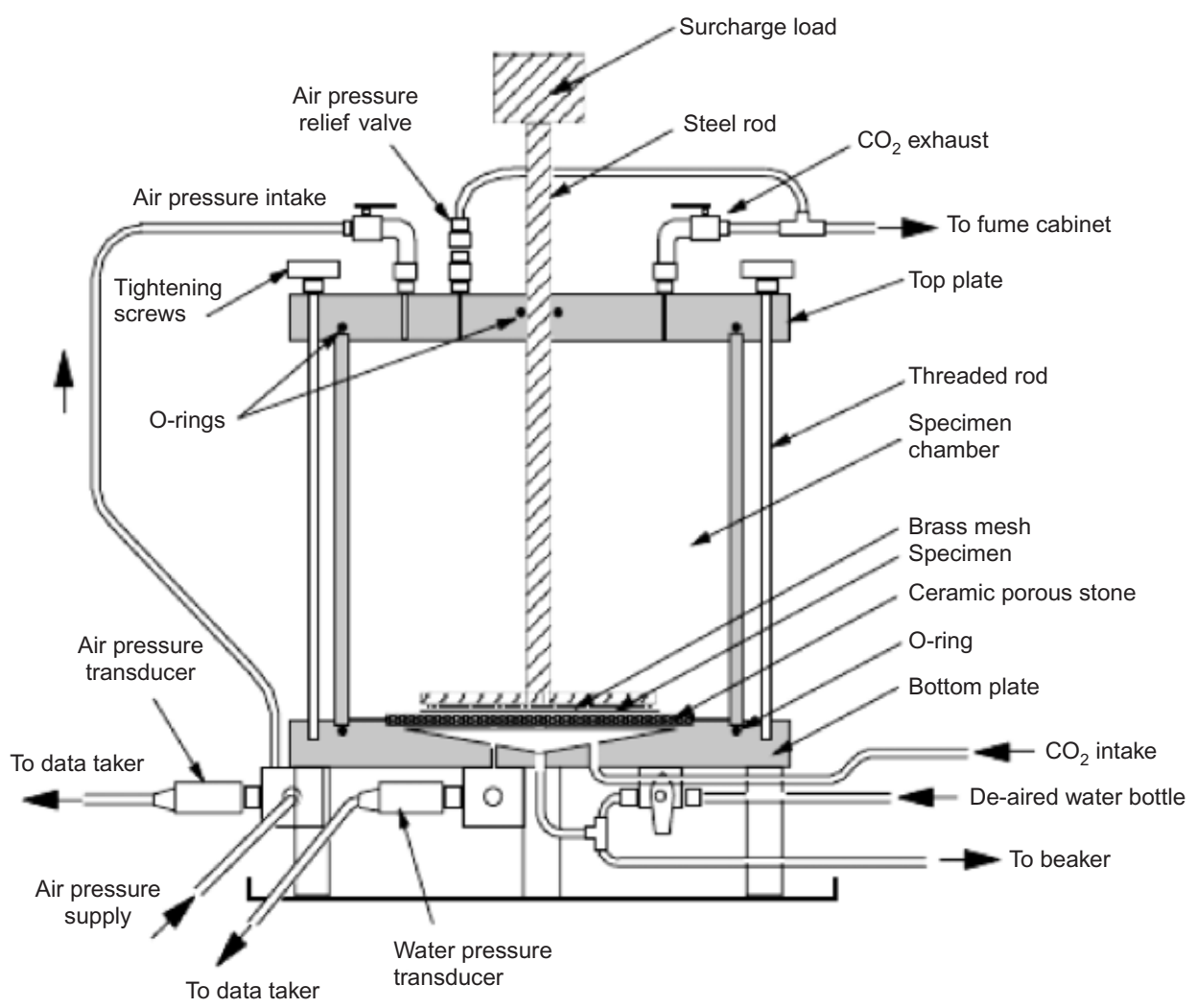

(b)

Figure 6. Pressure plate test: (a) conventional test used for soil specimens; (b) modified test used for geotextile specimens (Nahlawi et al. 2007a)



Figure 7. Conventional methods to determine the soil WRC using saturated salt solutions

$1500 \mathrm{kPa})$, and thermodynamic techniques are used for higher suctions. Thermodynamic techniques have not been used for geosynthetics, probably because the water content of geotextiles at high suction values is so low that its measurement has not been needed for practical applications.

A technique not used for characterization of the WRC of soils, but which has proven useful for characterization of geotextiles, is the capillary raise test. Specifically, Henry and Holtz (2001) monitored water capillary rise by submerging one end of a strip of geotextile in water and measuring the height to which the water rose. The height of capillary rise provides a good estimate of the water entry suction of the material in the in-plane direction (Stormont and Ramos, 2004; Nahlawi et al. 2008). A modification of this technique was presented by Lafleur et al. (2000), who measured the in-plane water retention function by submerging the end of a $500 \mathrm{~mm}$-long geotextile specimen strip in water and allowing it to 
equilibrate over $72 \mathrm{~h}$. The volumetric water content was measured at different positions above the water surface by cutting the specimen into 20 or $50 \mathrm{~mm}$-long segments and weighing the samples before and after oven-drying. Variations of this testing approach are discussed by Stormont and Ramos (2004), Krisdani et al. (2006), and Nahlawi et al. (2008). The test setup as presented by Lafleur et al. (2000) is shown in Figure 8. In-plane drying tests have also been conducted using initially saturated geotextile strips that are allowed to drain vertically under gravity, thus allowing the development of the in-plane WRC of geotextile in both wetting and drying paths (Nahlawi 2009).

Conventional techniques to define the WRC of geomaterials often require significant time to obtain limited data. For example, determination of the WRC for a highplasticity clay specimen may take several months. Also, conventional testing methods require the use of several specimens and destructive measurement of water content. Problems specific to WRC determination involve diffusion of air across porous ceramics, lack of control of volume change during drying and wetting (e.g. Cabral et al. 2004), and inability to impose a stress state representative of field conditions. Centrifugation has been used to alleviate the shortcomings of conventional characterization of the WRC. Centrifugation increases the body forces on a porous medium, accelerating fluid flow, because time increases quadratically with $g$-level. Centrifuges were first used to define the WRC by soil scientists and petroleum engineers in the early 1930s (Gardner 1937; Hassler and



Figure 8. Test apparatus used to measure the in-plane water retention function of unsaturated geotextile specimens (Lafleur et al. 2000)
Brunner 1945). Centrifuge technology has been recently used at the University of Texas at Austin for expeditious characterization of the unsaturated hydraulic properties of soils, and can provide insight into the unsaturated behavior of geosynthetics (McCartney and Zornberg 2010b; Zornberg and McCartney 2010).

The WRC of geomaterials is typically quantified by fitting experimental data to power law, hyperbolic, or polynomial functions (Brooks and Corey 1964; van Genuchten 1980; Fredlund and Xing 1994). Although the Brooks and Corey (1964) model is able to represent a sharp air-entry suction, the van Genuchten (1980) model has been most commonly used in numerical analyses, because it is differentiable for the full suction range. The van Genuchten (1980) model is given by

$$
\theta=\theta_{\mathrm{r}}+\left(\theta_{\mathrm{s}}-\theta_{\mathrm{r}}\right)\left[1+(\alpha \psi)^{N}\right]^{-(1-1 / N)}
$$

where $\theta_{\mathrm{r}}$ is the residual water content, $\theta_{\mathrm{s}}$ is the saturated water content (porosity), and $\alpha$ (units of $\mathrm{kPa}^{-1}$ ) and $N$ (dimensionless) are fitting parameters. Preliminary estimates of the WRC have been obtained using databases that rely on the granulometric distribution of soils (Fredlund and Xing 1994). The functions used to fit experimental data from WRC have also been proven to be useful for the case of geotextiles (Bouazza et al. 2006a; Nahlawi et al. 2007a).

\subsection{Hydraulic conductivity function of unsaturated geomaterials}

The relationship between hydraulic conductivity and suction, also referred to as the $K$-function, provides a measure of the increased impedance to water flow with decreasing water content. The saturated hydraulic conductivity $K_{\mathrm{S}}$ corresponds to the minimum impedance to water flow through geomaterials. Figure 9 shows the $K$-functions of different geomaterials. Near saturation, the coarser materials (sand and geotextile) have a comparatively higher hydraulic conductivity than the fine-grained materials (silt and clay). However, as the water content decreases, the coarser materials end up being less conductive than the fine-grained soil. Since the fine-grained materials can retain more water in the pores as suction increases, they



Figure 9. Typical $K$-functions for different geotechnical materials (after McCartney et al. 2005) 
have more pathways available for water flow and are thus more conductive than coarser materials. The good performance in arid climates of evapotranspirative covers relative to compacted clay covers has been attributed to the lower unsaturated hydraulic conductivity of the selected cover soils.

Conventional methods used to define the $K$-function may be costly, time consuming, and prone to error due to experimental issues involved in the control of water flow through unsaturated geomaterials. Accordingly, $K$-functions (e.g. such as those in Figure 9) are often predicted based on the information obtained using theoretical derivations based on the measured WRC. Specifically, the $K$ function obtained using the parameters from the van Genuchten-Mualem model (van Genuchten 1980) is

$$
K(\theta)=K_{\mathrm{s}} \sqrt{\frac{\theta-\theta_{\mathrm{r}}}{\theta_{\mathrm{s}}-\theta_{\mathrm{r}}}}\left\{1-\left[1-\left(\frac{\theta-\theta_{\mathrm{r}}}{\theta_{\mathrm{s}}-\theta_{\mathrm{r}}}\right)^{1-N}\right]^{1(1-N)}\right\}^{2}
$$

$K(\psi)$ can be defined by substituting $\theta$ from Equation 6 into Equation 7. Other predictive relationships for the $K$ function are given by Burdine (1953), Brooks and Corey (1964) and Fredlund and Xing (1994), among others. Nahlawi et al. (2007a) noted that the $K$-functions were better estimated by the van Genuchten WRC equation, because it is continuous.

In spite of the experimental difficulties, a few techniques have been proposed for direct determination of the $K$-function of soils in the laboratory (Benson and Gribb 1997). Techniques that have been used to measure the $K$ function of soil specimens typically involve flow of water through a specimen confined within a permeameter. The hydraulic conductivity can be measured either by applying flow across the specimen and measuring the corresponding hydraulic gradient, or by applying a hydraulic gradient and measuring the corresponding flow rate. Flow is applied to unsaturated soil specimens using surface infiltration imposed with flow pumps, lowering or raising of a water table to cause imbibition or drainage of water from a dry or saturated soil specimen, or by applying pressure to high-air-entry porous discs that transmit only water (not air). Figure 10a shows a typical permeameter setup used to measure the hydraulic conductivity using infiltration or evaporation (Meerdink et al. 1996; McCartney et al. 2007), and Figure 10b shows a typical permeameter used to measure hydraulic conductivity using cross-plane water flow in a flexible wall permeameter (Znidarcic et al. 1991; Lu and Likos 2005). Permeameters have differed in specimen confinement and size, control of boundary conditions, and availability of instrumentation. The $K$ function can be estimated using steady or transient flow processes. During steady water infiltration, a unit hydraulic gradient (e.g. $i=1$ ) is typically observed in the soil profile sufficiently far from a water table boundary. Accordingly, suction does not change with depth, and water flow is driven only by gravity. In this case, the hydraulic conductivity equals the imposed steady-state discharge velocity. Additional points of the $K$-function can

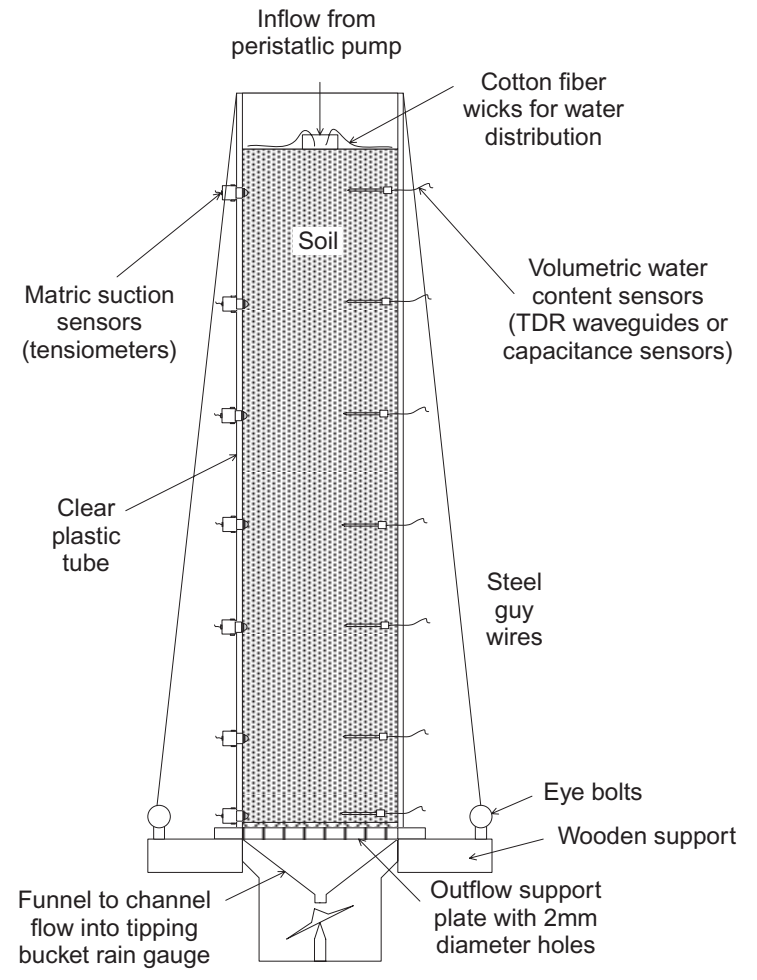

(a)

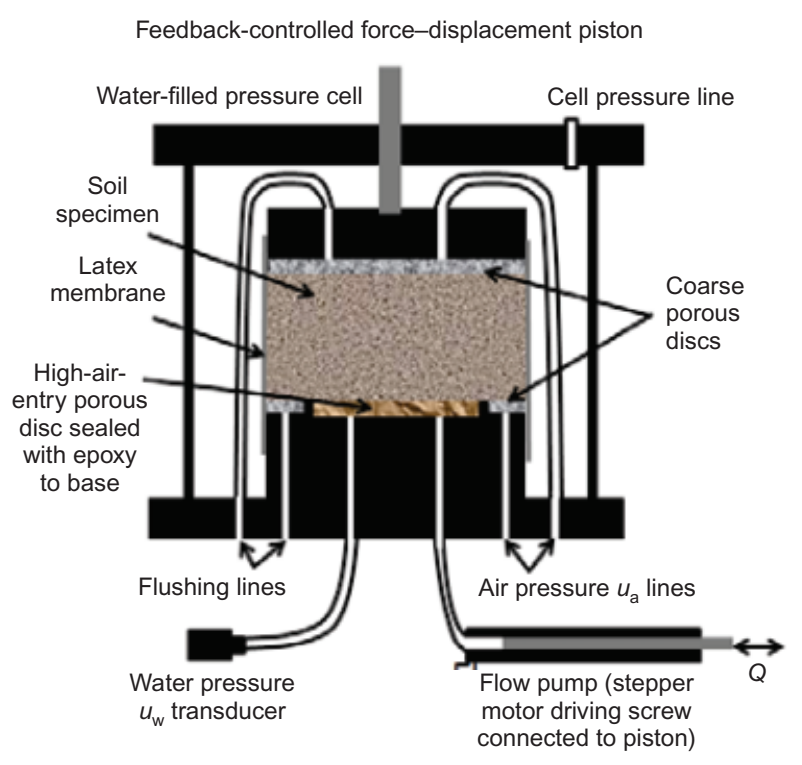

(b)

Figure 10. Permeameters for unsaturated flow testing: (a) column permeameter (McCartney and Zornberg 2010a); (b) flexible-wall permeameter (Znidarcic et al. 1991)

be obtained by changing the imposed flow. During transient flow processes, the suction and water content profiles are measured as a function of depth and time, and the $K$-function can be estimated using outflow measurements (Gardner 1956) or the instantaneous profile method (Watson 1996; Meerdink et al. 1996). While techniques based on transient processes yield more information about the $K$-function, steady-state techniques typically provide more reliable information (McCartney et al. 2007). 
To alleviate the difficulties associated with direct determination of the $K$-function of unsaturated soils, centrifuge testing has been used to define the $K$-function of soil used in projects involving the design of alternative covers (Nimmo et al. 1987; Conca and Wright 1992; Zornberg et al. 2003). For steady-state conditions, Darcy's law under increased gravitational field and an imposed discharge velocity can be directly used to determine the $K$-function, as follows:

$$
K(\psi)=-v /\left(-\frac{1}{\rho_{\mathrm{w}} g} \frac{\mathrm{d} \psi}{\mathrm{d} z}-\frac{\omega^{2} r}{g}\right)
$$

where $v$ is the imposed discharge velocity, $\omega$ is the angular velocity, and $r$ is the centrifuge radius. Points on the $K$-function are defined using Equation 8 after steadystate conditions are reached. Early centrifuge studies (Nimmo et al. 1987; Conca and Wright 1992) did not allow direct monitoring in-flight of the relevant variables (suction, water, discharge velocity) during testing. If the suction gradient in Equation 8 is assumed to be negligible, the hydraulic conductivity becomes inversely proportional to $\omega^{2}$. To alleviate the shortcomings of early studies, an improved centrifuge device was recently developed (Zornberg and McCartney 2010). This device incorporates the use of a low-flow hydraulic permeameter and a high- $g$ centrifuge capable of continuously, non-destructively, and non-intrusively measuring suction, water content, and fluid flow rate in a single specimen during centrifugation. Accordingly, the new centrifuge allows an expedited determination of both the WRC and the $K$-function from a single soil specimen in a single test. Figure 11a shows the centrifuge permeameter and its instrumentation layout, and Figure $11 \mathrm{~b}$ shows a view of the new centrifuge. A special low-flow fluid union is used to supply fluid from the stationary environment to the rotating specimen within the centrifuge. An important feature to point out is that the centrifuge permeameter shown in Figure 11a is essentially a column test such as that shown in Figure 10a. The centrifuge is a suitable tool to provide expeditious evaluation of the soil-geosynthetic interaction arising from a capillary break induced by geotextiles.

The $K$-function of nonwoven geotextiles has been measured in only a few studies. McCartney et al. (2008) adapted a hanging column test to measure outflow (Figure $12 a$ ), and used it to calculate the $K$-function of unsaturated geotextiles. Specifically, a Mariotte burette was used to maintain a constant suction value on the geotextile specimen, while still permitting outflow to be measured with the burette. This study used an approach proposed by Gardner (1956) to calculate the hydraulic conductivity from the outflow data obtained during different increments of suction applied with the hanging column. The measured $K$-function was consistent with the $K$-function predicted using the WRC. The hydraulic conductivity of geotextiles has also been estimated using instant profiling methods (e.g. Morris 2000; Stormont and Morris 2000). Figure 12b shows a view of the instantaneous profile setup. Very fine wire probes were used for time domain reflectometry (TDR) measurements to minimize their impact on water



(a)



(b)

Figure 11. Permeameter for unsaturated soils: (a) permeameter; (b) centrifuge layout including a permeameter

movement through the geotextile. However, these tests required a long time, and interpretation of transient flow using the instant profiling method, and variability in the shape of the $K$-function is often obtained. Stacked geotextile and capillary rise tests have also been used to determine the hydraulic conductivity of unsaturated geotextiles (Knight and Kotha 2001; Nahlawi et al. 2008). In principle, it is possible to determine the $K$-function concurrently with tests used to measure the WRC, but difficulties arise as a result of variability in results, head losses through the high-air-entry porous stones, and the lack of instrumentation in the stacked geotextile and capillary rise tests. 


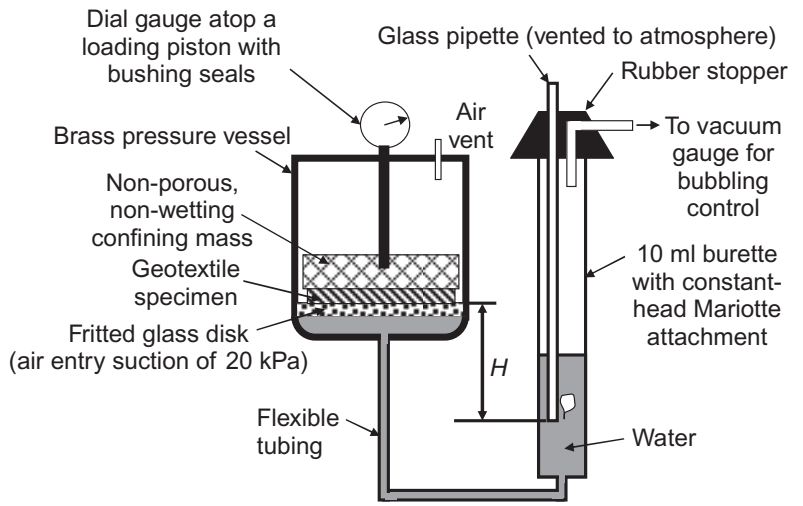

(a)

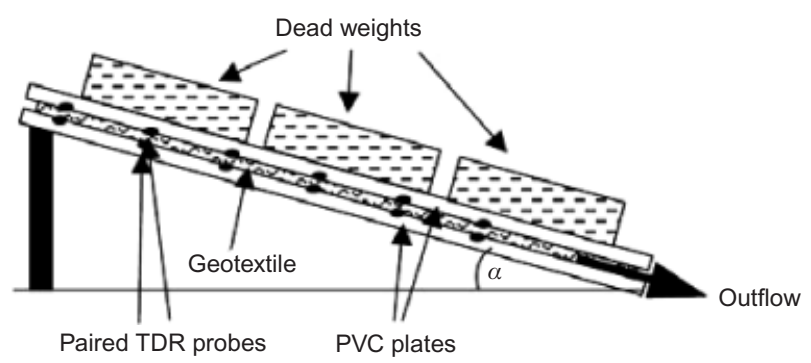

(b)

Figure 12. Equipment to measure the hydraulic conductivity of unsaturated geotextiles: (a) hanging column with outflow measurement; (b) inclined instantaneous profile test (Morris 2000)

\section{ALTERNATIVE COVER SYSTEMS}

Various types of alternative cover have recently been implemented in arid and semi-arid climates. As shown in Figure $1 b$, both evapotranspiration and water storage are relevant water balance components that can benefit the performance of alternative covers. The focus of this paper is on capillary barriers, but the various types of alternative cover are discussed next as they are closely related, and can all involve geosynthetic components.

\subsection{Monolithic covers}

Monolithic covers are evapotranspirative covers that consist of a single soil layer placed directly over the waste (Zornberg et al. 2003). Figure 13a shows a schematic view of a monolithic soil cover. The soil layer acts both as a substrate for vegetation and as a hydraulic barrier. A foundation layer consisting of the same soil type is typically used to provide a level surface above the waste. Early research focused on investigation of the long-term behavior of natural soil layers in arid regions, assuming that the behavior is analogous to that of an engineered monolithic cover (Waugh et al. 1994). These studies found that water content fluctuations in natural analogues in recent geologic history are typically confined to the upper meters of soil, indicating that monolithic covers may be an acceptable long-term solution to waste disposal.

Design of monolithic covers requires proper characterization of the hydraulic properties ( $K$-function and WRC) of the soils, as well as determination of the appropriate thickness of the engineered soil cover. Figure 13b shows

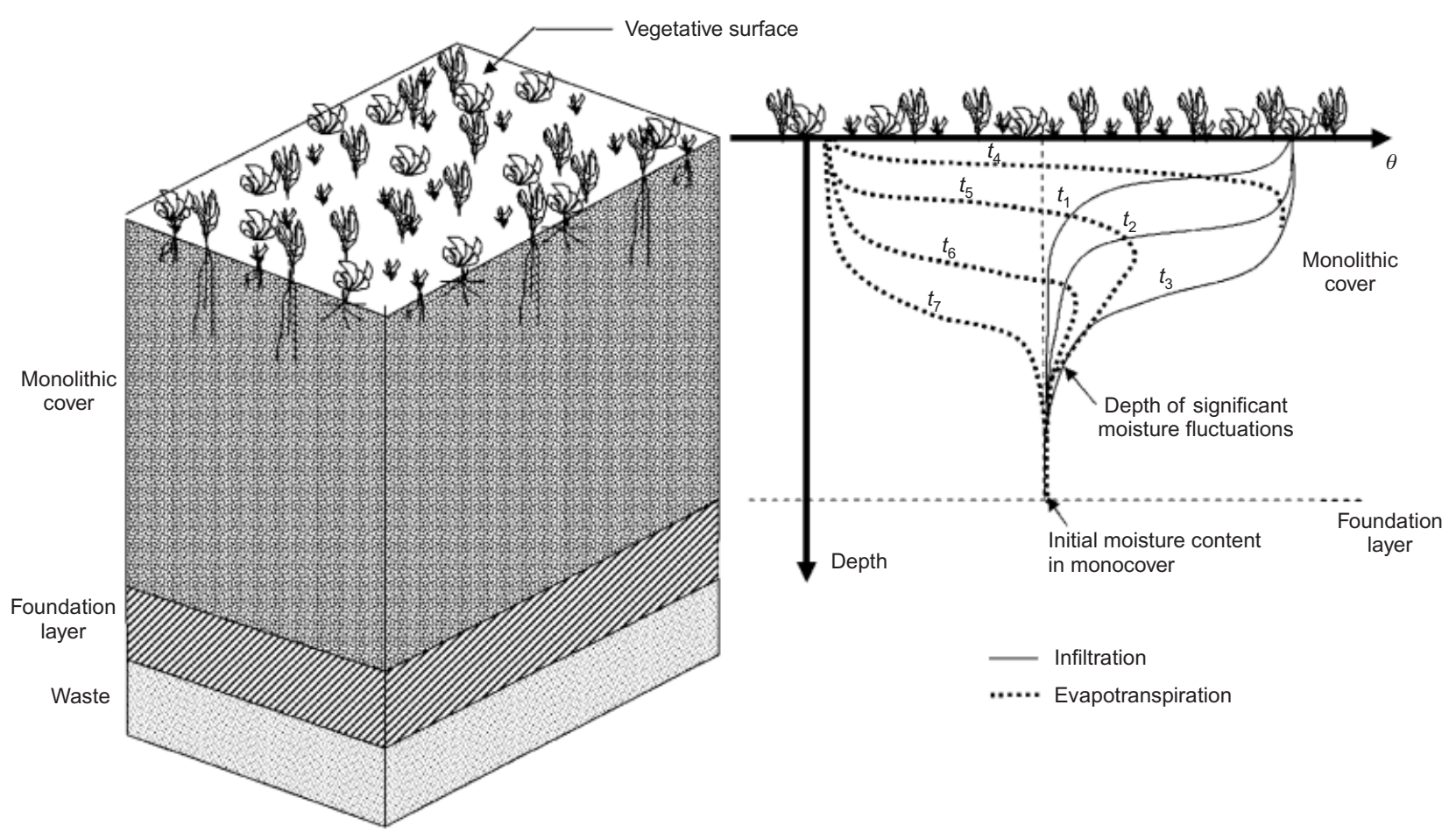

(a)

(b)

Figure 13. Monolithic cover: (a) soil profile; (b) typical seasonal water content fluctuations (Zornberg and McCartney 2007) 
schematic water profiles, illustrating typical seasonal fluctuations in a properly performing monolithic cover. The water profiles illustrate wetting during infiltration events and subsequent drying due to evapotranspiration. Although some water fluctuations are expected to reach the base of the cover in extreme events, most of the water fluctuations are expected to be confined within the upper portion of the cover. Monolithic cover design requires selection of the cover thickness and soil-water storage necessary to keep the basal percolation below a minimum allowable (design) value, given the expected weather conditions for a site.

The cover storage depends on the shape of the soil WRC. A parameter that has been used to quantify the water storage is the field capacity, which is defined as the threshold water content value above which the soil no longer retains water by capillarity under the effects of gravity (Zornberg et al. 1999). When water is added to a soil that is at field capacity, drainage occurs. The field capacity may be obtained from infiltration tests, although a generally accepted value for silt and low-plasticity clay soils is the water content corresponding to a suction of $33 \mathrm{kPa}$ (Nachabe 1998; Meyer and Gee 1999). A preliminary measure of the storage capacity of a monolithic cover can be established by multiplying the volumetric water content at field capacity (obtained using the WRC) by the cover thickness.

\subsection{Capillary barrier covers}

Capillary barriers are evapotranspirative covers composed of a layered system that involves a fine-grained soil (silt, clay) placed over a coarse-grained geomaterial (sand, gravel, nonwoven geotextile). Capillary barriers use the contrast in hydraulic properties between the fine- and coarse-grained soils to enhance the ability of the finegrained material to store water (Shackelford et al. 1994; Stormont and Anderson 1999; Khire et al. 1995, 2000). The upper portion of this cover can be considered as a monolithic cover (Section 4.1) that benefits from added storage induced by a capillary break. The capillary break minimizes the amount of water that can flow across the interface from the fine-grained component of the cover into the coarse geomaterial until the overlying soil is nearly saturated. The pressure (or energy) in the water within the fine-grained soil must increase (i.e. the suction must decrease) to the point where it is sufficient to overcome the surface tension forces holding the water within the small pores. The pressure (or energy) in the water also has to be sufficient to force the air out of the pores of the coarse-grained soil (or the geotextile). The energy in the pore water in an unsaturated soil can be quantified by the matric suction (see Equation 1), equal to the difference between the pore air and water pressures. Figures $14 \mathrm{a}$ and $14 \mathrm{~b}$ show WRCs and $K$-functions for a sand or nonwoven geotextile (coarsegrained component), and a low-plasticity clay (finegrained component).

The capillary break concept relies on the continuity of suction, even at the interface between two different materials. Accordingly, the shapes of the WRCs for the soil and geotextile indicate that the degree of saturation in the two materials may be different for the same suction. For example, Figure 14a shows that when a system is at an initial suction of $10 \mathrm{kPa}$, the clay has a degree of saturation of 0.7 (relatively wet) while the underlying coarse material is at a residual water content of 0.03 (very dry). Figure $14 \mathrm{~b}$ indicates that at this suction of $10 \mathrm{kPa}$ the clay has a hydraulic conductivity of approximately $1 \times 10^{-9} \mathrm{~m} / \mathrm{s}$, while the sand has considerably higher impedance to flow (it is essentially impervious). Consequently, if water infiltrates into the fine-grained material after a precipitation event and reaches the interface with the coarse-grained material, it can progress into the coarse-grained material only at a very slow rate. That is, water will accumulate at the interface until the suction at the interface reaches a value at which the hydraulic conductivity of the coarse-grained material is no longer below that of the fine-grained material $(4.0 \mathrm{kPa}$ in Figure $14 \mathrm{~b})$. This suction value is referred to as the breakthrough suction. A breakthrough suction of $4.0 \mathrm{kPa}$ is significantly below the suction corresponding to field capacity (typically considered at $33 \mathrm{kPa}$ for clay), which indicates that the degree of saturation in the clay will be relatively high (95\%) when breakthrough occurs. For suction values less than that corresponding to field capacity, water would have drained downwards by gravity had the capillary break not been present. When the breakthrough suction is reached, leakage is observed into the coarse-grained layer at a rate approaching that corresponding to the saturated hydraulic conductivity of the barrier layer.

Figure $14 \mathrm{c}$ shows a schematic view of a capillary barrier. Similar to the monolithic cover, the soil layer acts both as a substrate for vegetation and as a hydraulic barrier (or capillary retention layer, CRL). However, a coarse-grained material (capillary break layer, CBL) is placed over the foundation material to create a capillary break at the interface between the CRL and the CBL). Figure $14 \mathrm{~d}$ shows schematic water profiles illustrating the expected seasonal fluctuations in a properly performing capillary break cover. Unlike the monolithic cover, the water front can reach the bottom of the CRL layer without resulting in basal percolation, provided the water at the interface does not exceed the breakthrough value. An important benefit of capillary breaks is that the water storage within the fine-grained soil can exceed its freely draining state (field capacity). Consequently, more water can be stored in a capillary break cover than in a monolithic cover of equivalent thickness. Alternatively, a thinner fine-grained soil layer can be used in a capillary break cover to obtain the same water storage as in a monolithic cover. Inclined covers may experience lateral diversion, which leads to greater water contents in the lower portion of a slope. Conservatism should be used in capillary barrier design, as these barriers have typically been reported to experience breakthrough during spring snowmelt when plant evapotranspiration is at a minimum (Khire et al. 1999). In addition, preferential flow through larger pores may lead to significant variability in the breakthrough suction (Kampf and Holfelder 1999). 


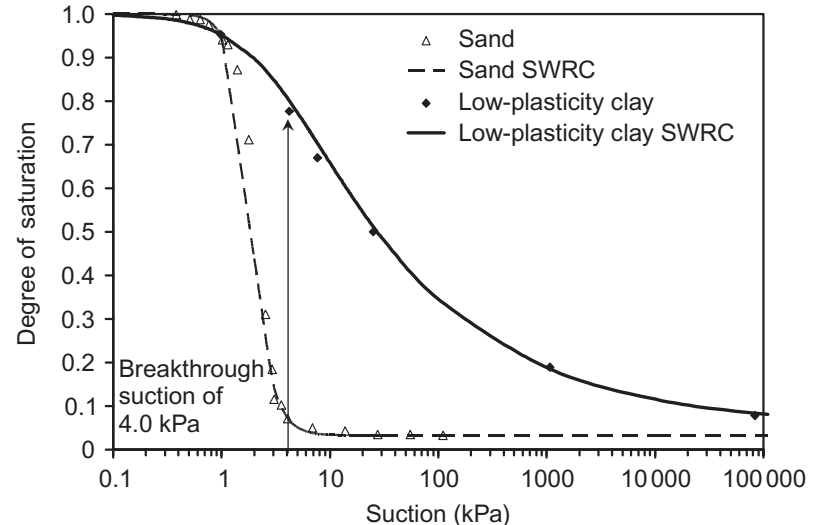

(a)

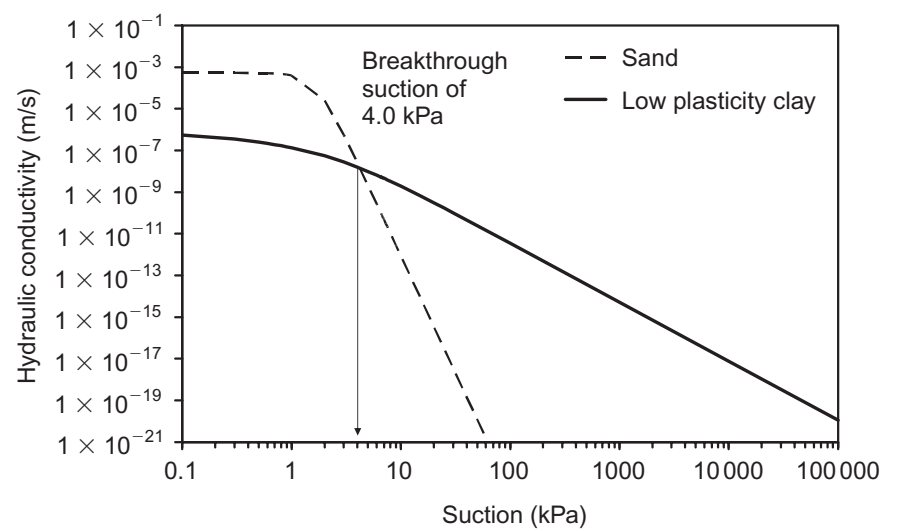

(b)

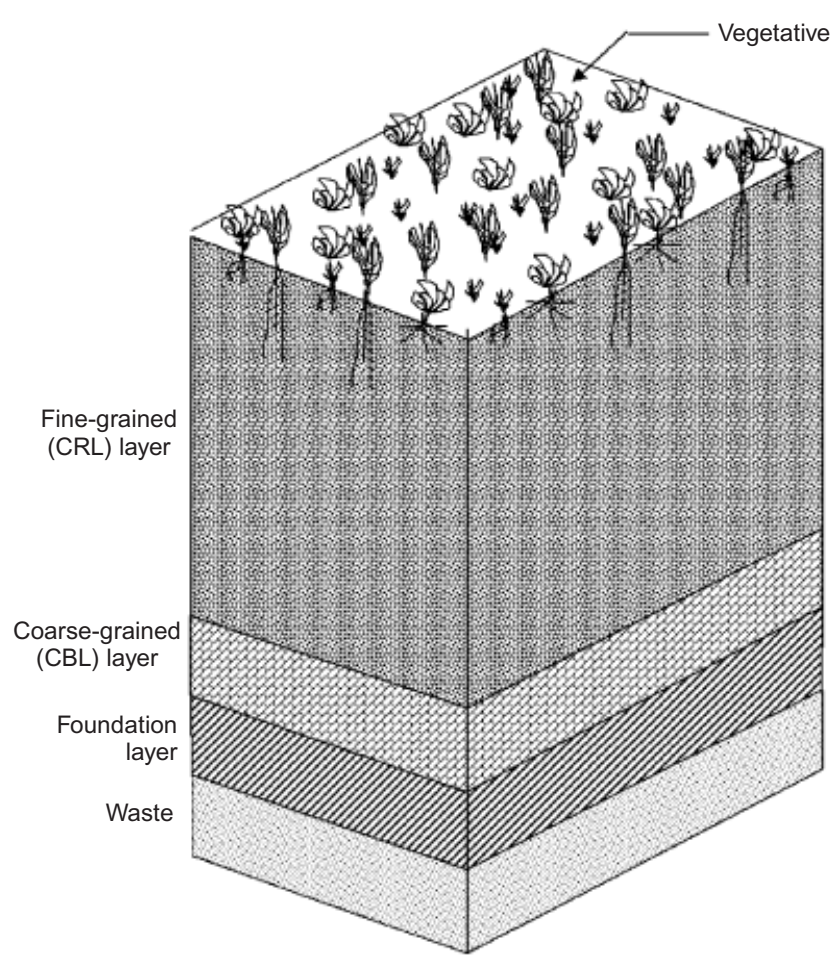

(c)

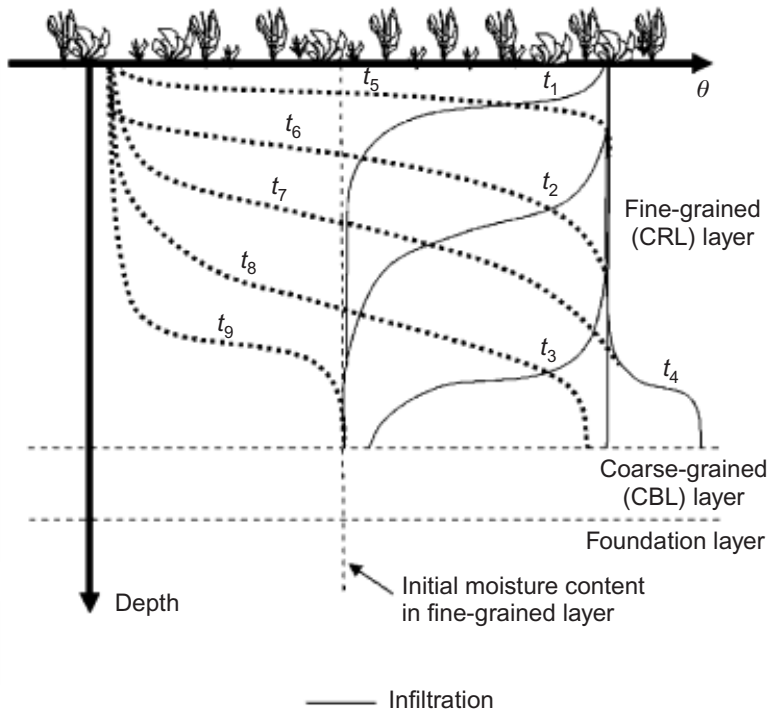

........ Evapotranspiration

(d)

Figure 14. Capillary barrier details: (a) conceptual SWRC illustration of the capillary break effect; (b) conceptual $K$-function illustration of the capillary break effect; (c) capillary break soil profile; (d) typical seasonal water content fluctuations (Zornberg and McCartney 2007)

\subsection{Anisotropic barrier covers}

Anisotropic barriers are similar to capillary barriers, but their design accounts for the internal lateral drainage through one or more drainage layers resulting from the inclination of the cover (Stormont 1995; Bussiere et al. 2003; Parent and Cabral 2005). Figure 15a shows a schematic view of an anisotropic barrier. Anisotropic layers typically involve a soil vegetation substrate overlying a coarse-grained drainage layer, which are in turn underlain by a primary barrier layer and a second coarsegrained layer to provide a capillary break. The second coarse-grained drainage layer functions both as a capillary break to the vegetation substrate and as a drainage layer for breakthrough water. Figure $15 \mathrm{~b}$ shows water profiles illustrating the expected trends along the length of an anisotropic barrier during a wet season. The capillary break at the drainage layer increases the water storage capacity of the vegetative substrate. Infiltration of water into the barrier layer generally occurs towards the toe of the slope as a result of water accumulation from infiltration and lateral drainage. Field comparisons between the performance of test-scale capillary break, anisotropic and monolithic covers performed by Dwyer (1998) indicate that the anisotropic cover performed well compared with the other covers for the same weather conditions over a 5-year monitoring program. However, its construction was the most difficult of the three covers, owing to the multiple soil layers. 




(a)

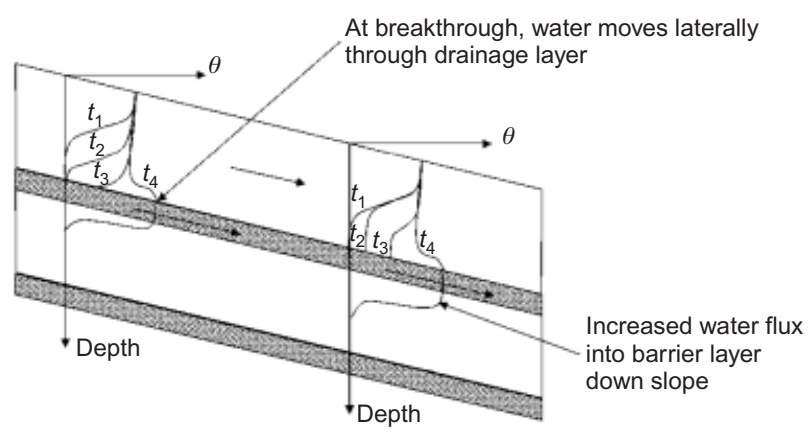

(b)

Figure 15. Anisotropic barrier: (a) soil profile; (b) typical water content fluctuations during infiltration (Zornberg and McCartney 2007)

\section{COLUMN STUDIES}

Column studies have been conducted with the objective of reproducing the behavior of geosynthetic capillary barriers under controlled laboratory conditions. These studies have been used to evaluate water flow across layered geomaterials under unsaturated conditions (Stormont and Anderson 1999), and to determine the hydraulic conductivity of unsaturated soil layers (Moore 1939; McCartney et al. 2007). Several column tests have been reported in which constant infiltration rates were applied to a clay layer underlain by a nonwoven geotextile (McCartney et al. 2005; Stormont et al. 2008; McCartney and Zornberg 2010a). Other tests have been conducted by inducing a constant head by ponding water atop the soil surface (Bathurst et al. 2007, 2009). For a constant-head inflow condition, a saturated wetting front passes through the soil, whereas for a constant flow rate condition (with a flow rate less than the saturated hydraulic conductivity), an unsaturated wetting front passes through the soil column. The infiltration condition involving evaluation of an advancing unsaturated wetting front is of particular relevance for the review presented in this paper, as it corresponds to conditions representative of alternative cover systems.

The changes in water content with time in a column study conducted at the University of Texas at Austin to evaluate the development of a capillary barrier effect are shown in Figure 16. Water was supplied to the top surface of soil at a constant inflow rate, and its transient infiltration through the soil column was monitored both visually



(a)

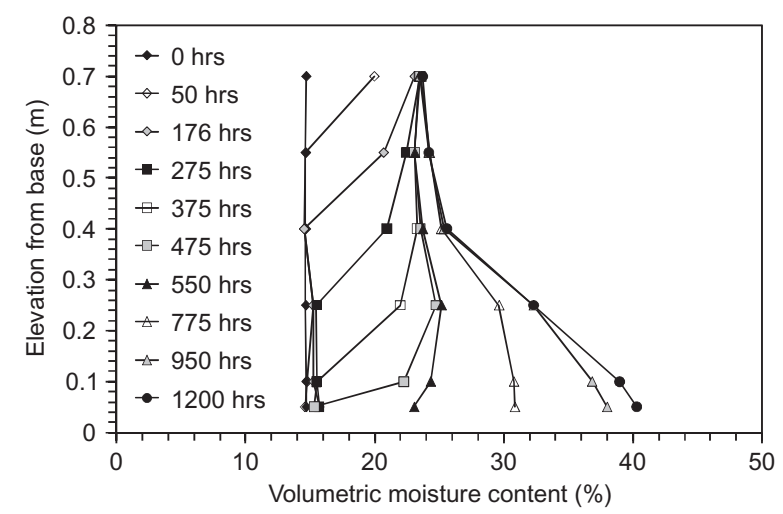

(b)

Figure 16. Results from column infiltration tests: (a) TDR water content time series for different heights from the base of a soil layer undergoing infiltration from the surface at a constant rate; (b) water content profiles with depth for different times

and using embedded water content sensors. Specifically, the water content was inferred using time domain reflectometry (TDR) waveguides placed at different elevations on a $750 \mathrm{~mm}$-long column of low-plasticity clay underlain by a geocomposite. As illustrated by the water content data shown in Figure 16a, three distinct phases of water flow can be identified for a constant infiltration rate of $4 \times 10^{-8} \mathrm{~m} / \mathrm{s}$ (approximately 100 times smaller than the saturated hydraulic conductivity of the soil). Initially, the entire profile was relatively dry, with an as-compacted volumetric water content of $15 \%$. Although the infiltration rate supplied at the top of the profile is constant, the wetting front moves through soil layer as a transient process. As the wetting front reaches the location of each of the TDR waveguides the water content is observed to increase, up to a value of approximately $24 \%$. Once the wetting front reached the base of the soil layer $(550 \mathrm{~h})$, water did not immediately flow into the geotextile. Instead, because of the capillary break, water accumulated within the soil immediately above the geotextile until the matric suction was reduced to a value at which capillary breakthrough could occur. Specifically, outflow was collected from the base of the column only once the soil reached a water content of approximately $40 \%$ (degree of saturation of $90 \%$ ). The breakthrough suction is consistent with the suction value expected based on the WRCs for these materials (see discussion in Section 4.2). Once 
outflow was collected after breakthrough, steady downward flow of water was established through the soilgeotextile system.

Figure 16b shows the water content profiles with height at different times. The results in this figure illustrate better the impact of the geosynthetic capillary break on the water storage within the soil layer during infiltration at a constant flow rate. The water content towards the top of the profile $(\theta=25 \%)$ corresponds to the condition in the soil layer in which there is no impact of the bottom boundary condition (i.e. a profile without the influence of a capillary barrier). This particular value of water content corresponds to the infiltration rate used in this study, assuming that infiltration occurs under a unit hydraulic gradient. During infiltration under a unit hydraulic gradient the suction does not change with height, so the total head difference with height equals the elevation head change with height. If the suction is constant with height, this implies that the water content should also be constant with height. A constant water content value with height is noted only in the top of the column for all times and for early stages of infiltration before the wetting front reached the level of the geosynthetic (i.e. before $550 \mathrm{~h}$ ). The water content at the base of the soil layer continued to increase after the wetting front had reached the level of the geosynthetic, beyond the water content corresponding to infiltration under a unit hydraulic gradient. Specifically, as shown in the figure, water content increases to a value of approximately $40 \%$ as a result of the development of a capillary break. As also shown in Figure 16b, approximately $0.3 \mathrm{~m}$ of soil experienced an increase in water storage due to the capillary break above that corresponding to infiltration under a unit hydraulic gradient. Additional column tests having greater lengths and different geosynthetic characteristics reported by McCartney and Zornberg (2010a) indicate that the geosynthetic capillary barrier can lead to an increase in soil-water storage up to a height of $0.5 \mathrm{~m}$ above the geosynthetic.

A discussion of the increase in water storage in a soil layer due to a capillary barrier must acknowledge the effect of the infiltration rate applied to the soil surface. The data in Figure 17 show the progression in water

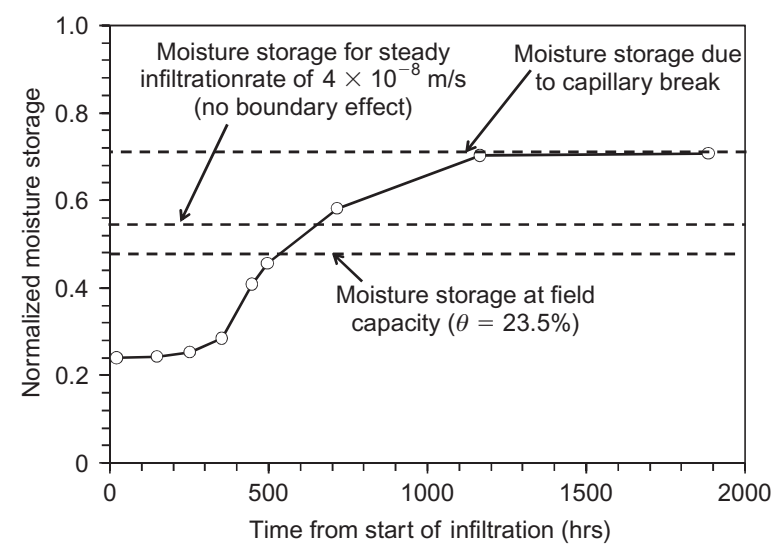

Figure 17. Increase in normalized water storage in the $300 \mathrm{~mm}$ of soil overlying the capillary break with time from the start of infiltration storage in the soil with time during infiltration, calculated by integrating the water content profiles in Figure $16 \mathrm{~b}$ from 0 to $300 \mathrm{~mm}$. The water storage in this figure was normalized in relation to the water storage at saturation (i.e. the maximum water storage). Also shown in this figure are the water storage values expected in the soil layer for different infiltration rates. The water storage was also higher than that corresponding to the 'unit-gradient' infiltration into a soil profile with no lower boundary effects, in which case the water content depends only on the magnitude of the infiltration rate. Further, the water storage of the soil layer at capillary breakthrough is significantly higher than the field capacity water storage. The field capacity water storage is an empirically derived quantity, which generally reflects the volume of water that can be stored in a soil against the downward pull of gravity.

Theoretical investigations have described the reasons why water storage in the soil layer under steady-state infiltration and without the influence of a capillary barrier is directly related to the magnitude of the infiltration rate (Choo and Yanful 2000; Dell'Avanzi et al. 2004). McCartney et al. (2005) found that the use of a constant inflow rate smaller than $K_{\mathrm{s}}$ led to a $40 \%$ increase in water storage near the geosynthetic capillary in relation to storage that occurs without a capillary barrier. On the other hand, if the inflow rate equals $K_{\mathrm{s}}$, no increase in water storage should be expected beyond that occurring in the soil during infiltration. This is because infiltration is occurring under saturated conditions, so the water storage in the soil during infiltration equals the maximum water storage in the soil. This response was confirmed by Bathurst et al. (2007), who performed infiltration tests on soil-geotextile profiles in which infiltration was imposed by ponding water on the soil surface, and found that capillary breakthrough occurred when the wetting front (with a suction value of approximately $0.0 \mathrm{kPa}$ ) reached the geosynthetic interface. Bathurst et al. (2009) and Siemens and Bathurst (2010) provided further information and numerical analysis from this testing program, including transient suction and water content profiles with time. These results confirm that the geotextile led to only a short delay in the progress of the saturated wetting front through the soil layer. This finding implies that an increase in volumetric water content is not expected because of the capillary break effect for infiltration conducted under ponding conditions. Although the results of this study are relevant for many practical applications, the occurrence of sustained ponding is not a representative condition for the design of alternative cover systems, which have been typically used in arid and semi-arid conditions.

Additional column studies have corroborated the previously discussed response of capillary barriers involving geosynthetics. Stormont and Morris (2000) carried out infiltration column tests using a silty sand layer placed over a layer of coarse sand or silty sand. A control test and a test with a nonwoven polypropylene geotextile layer at the interface between soil layers were reported. Suction was measured using tensiometers placed directly above 
and below the interface location. They reported the development of a capillary break when the geotextile was placed between the silty sand and the underlying coarse sand. Krisdani et al. (2006) reported the results on a column fitted with tensiometers and TDRs to examine the saturated-unsaturated response of a geocomposite under infiltration simulating rainfall conditions. Both physical and numerical tests showed that the geosynthetic inclusion caused a break in the pore water pressure-head profile through the height of the fine sand column at the elevation of the inclusion during and after infiltration loading. Nahlawi et al. (2007b) carried out infiltration tests in a dry sand-geotextile column under constant head with the objective of determining the transient pore water pressure response and the advancement of the wetting front. The column was instrumented with air pressure transducers, waters probes, pore pressure and tensiometers. The test results showed the development of preferential flow (fingering) in the dry sand with the accumulation of water above the geotextile before the breakthrough.

Column studies have also been performed to quantify the impact of different variables on the behavior of capillary barrier systems. McCartney et al. (2007) performed a series of infiltration column tests on $125 \mathrm{~mm}$ long soil columns, and found that soil density may impact on the geosynthetic capillary barrier behavior. In particular, this study found that loosely compacted clays reach breakthrough at smaller suctions than highly compacted clays. However, the speed of the water front is significantly higher in loosely compacted soils, leading to a condition in which comparatively short precipitation events lead quickly to full saturation of the cover layer. McCartney and Zornberg (2010a) investigated the transient movement of water in unsaturated soil layers underlain by a geocomposite drainage layer (GDL) during application of cycles of infiltration and evaporation to the soil surface. A $1350 \mathrm{~mm}$-high soil column was used to evaluate the impact of the GDL on the vertical distribution of volumetric water content in the soil above the geosynthetic drainage layer during transient infiltration. Results from this test indicate that the capillary break effect influences the volumetric water content profile in the soil column up to a height of $500 \mathrm{~mm}$ above the GDL. Specifically, an increase in volumetric water content of up to $20 \%$ was observed above that expected for the case of infiltration under a unit hydraulic gradient. Because of the long duration of this test $(2000 \mathrm{~h})$, a shorter soil column with a height of $150 \mathrm{~mm}$ was also used to investigate the values of matric suction and volumetric water content near the soil/GDL interface during cycles of infiltration and evaporation. The measured suction and volumetric water content at breakthrough were consistent after each breakthrough event. The volumetric water content in the soil after each breakthrough event was found to be close to that corresponding to the matric suction where the dryingpath WRC of the nonwoven geotextile rapidly transitions from residual to saturated conditions. Further, and consistent with results reported by Stormont et al. (2008), the capillary break was re-established after the matric suction at the soil/geosynthetic interface had increased over the breakthrough suction value. This is important, because it indicates that geosynthetic capillary barriers constructed in the field are expected to have a long-term sustainable performance even if they experience capillary breakthrough.

\section{PRACTICAL APPLICATIONS INVOLVING CAPILLARY BARRIERS}

Although the main focus of this paper is on geosynthetic capillary barriers for alternative cover design, many other geotechnical and environmental applications can benefit from capillary barriers induced by nonwoven geotextiles. Along these same lines, there are also several situations in which poor performance of an earth structure involving nonwoven geotextiles may be encountered if the capillary break effect is ignored. A brief overview of these general geotechnical applications is presented in Section 6.1. The impact of geosynthetic capillary barriers in the specific case of alternative cover systems is discussed in Section 6.2 .

\subsection{Impact of geosynthetic capillary barrier in geotechnical applications}

\subsubsection{Impact on slope stability}

The capillary break effect may have detrimental implications for the performance of slopes or walls if a nonwoven geotextile is expected to drain water from an unsaturated fill in a similar way as if the fill were fully saturated. Richardson (1997) reported the failure of a slope with a geosynthetic underdrain. Specifically, while design calculations had used the dry unit weight of the soil in the stability analysis, the soil above the geosynthetic underdrain became nearly saturated as a result of the development of a capillary barrier. Consequently, the actual unit weight was indeed approaching that of a saturated fill. This resulted in a decrease in the factor of safety by a factor corresponding to the ratio of these two unit weight values (i.e. $1 /(1+w)$, where $w$ is the gravimetric water content).

The impact on slope stability induced by infiltration through soil-geosynthetic systems needs to account for the results of column studies (see Section 5). For example, results from the column study reported by Bathurst et al. (2009) indicate that for soil-geotextile systems subjected to cross-plane infiltration, the current design practice of requiring that the saturated hydraulic conductivity of the geotextile be at least equal to that of the overlying soil may need to be revised. Specifically, a higher ratio between the saturated hydraulic conductivity of the geotextile and that of the soil may be needed in order to provide an additional margin of safety, and minimize water ponding over the geotextile layer.

Iryo and Rowe (2005a) performed a series of transient finite element analyses of water infiltration into soilgeocomposite layers considering a variety of soil types, slope inclinations and infiltration rates. The influence of these variables on the effectiveness of geocomposites as a drainage material and as a capillary barrier indicated a higher flow into the geocomposite for decreasing slope 
angle and increasing infiltration rate. In other words, this study found that the impact of the geosynthetic capillary barrier is more pronounced for smaller infiltration rates and steeper slopes. This is consistent with the observation reported in Section 5 that the rate of infiltration affects the water storage of the soil above a geosynthetic capillary barrier. Modeling results confirmed the observation of Richardson (1997) that the soil immediately above the geocomposite becomes nearly saturated before the geocomposite starts draining water, and it remains under high water conditions over long periods of time after the infiltration event.

The theory of water flow in unsaturated geomaterials presented in Section 2 considered for simplicity that the pore air pressure equals zero. However, in landfill cover systems atop actively biodegrading waste, the pore air (or gas) pressure is positive. For example, Thiel (1999) reported that slope stability can be seriously affected by the gas pressure beneath a geomembrane liner. However, this condition may also be expected if geotextiles, rather than geomembranes, are used in the cover system and a capillary break develops at the geotextile interface. Since wet geomaterials have a much lower permeability to gas flow (Bouazza 2004), accumulation of water over the geotextile would act as a barrier to gas flow, as in the case of a geomembrane. This effect should be considered in the design of gas collection systems, even for the case of covers that do not include geomembranes.

\subsubsection{Impact on reinforced-soil structures}

Proper drainage within the backfill is one of the most important design issues to be considered in the design of reinforced-soil structures. This is especially the case in earth retention systems involving poorly draining backfills, because of their higher susceptibility to develop positive pore water pressures (Zornberg and Mitchell 1994; Mitchell and Zornberg 1995). Geosynthetic drains have been proposed for use within these backfill soils to reduce the drainage paths. However, it should be recognized that these drains will conduct water only after the soil has become nearly saturated. As in the previously discussed case of slope underdrains, the soil unit weight to be considered in the design should correspond to nearly saturated conditions because of the capillary break effect.

Iryo and Rowe (2005b) used finite elements to simulate the hydraulic and mechanical response of geosyntheticreinforced embankments. Specifically, numerical simulations were conducted to examine the effect of geotextile arrangement and infiltration conditions, and to assess the effectiveness of nonwoven geotextiles as drainage material. Using pore water pressures obtained from the finite element analysis, water flow analyses indicated that nonwoven geotextiles may retard the water flow in situations where the pore pressure is negative, whereas they act as a drainage material in situations where the pore pressure is positive. Some of the reported results are shown in Figure 18, showing that nonwoven geotextiles contributed more significantly as drains within the fill than as reinforcement inclusion. Garcia et al. (2007) reported experimental results that are useful to assess the observations made by
Iryo and Rowe (2005b) using numerical simulations. In this study, model embankments were built using two layers of permeable geosynthetics, and their performance was assessed under infiltration and evaporation conditions. Their results indicate that geosynthetics embedded within the soil approached saturation only when the pore water pressure within the surrounding soil approached zero. Local failure was observed during infiltration, caused by water accumulated above the geosynthetics. Failure occurred because of pore water pressure increases within the soil immediately above the geosynthetic layers. Garcia et al. (2007) observed that geotextiles placed in the form of strips minimized the capillary barrier effect and facilitated water drainage.

\subsubsection{Impact on pavements}

Two major issues that affect pavement performance are frost heave and capillary rise. Frost heave can lead to significant differential movements in the subgrade and base course layers of pavements, which can contribute to pavement surface cracking and deterioration. Capillary rise from fluctuating water tables can lead to a change in the stiffness of pavement soils. Specifically, compacted base course soils have high stiffness and shear strength because of the high matric suction induced under comparatively low water content values. As compacted fills are frequently used in flexible pavements and foundations for structures (walls, footings), decrease in suction induced by water flow into the fill may compromise the performance of the system. Nonwoven geotextiles could be used within the flexible pavement as a hydraulic barrier to capillary flow (from a water table or drainage ditch), as shown in Figure 19. Configurations such as these can be used to prevent water flow that could lead to reduction in stiffness, frost heave, or swelling of expansive clay subgrades.

Henry (1996) proposed the use of geotextiles as barriers to frost heave in pavement structures. Laboratory tests showed that properly selected geotextiles reduce frost heave in soils by functioning as capillary barriers. Christopher et al. (2000) described the results from a field experiment in Maine in which geocomposites were placed at different depths within the subgrade to reduce frost susceptibility in pavements. Henry and Holtz (2001) investigated the potential for geosynthetic capillary barriers to reduce frost heave in soils. In this study, soilgeosynthetic systems were subjected to freezing temperatures, and the frost heave and final water content profiles of specimens were measured. Isolated geotextile layers placed did not experience a reduction in frost heave beyond control specimens. However, geocomposites, comprising a drainage net sandwiched between two needlepunched polypropylene geotextiles, were found to reduce frost heave. The presence of the air gap within the drainage net was found to minimize the upward movement of water into the overlying soil layer, observed when using isolated geotextiles.

Clough and French (1982) performed an early study on the use of geotextiles in pavement systems to mitigate water fluctuations in base courses induced by capillary 


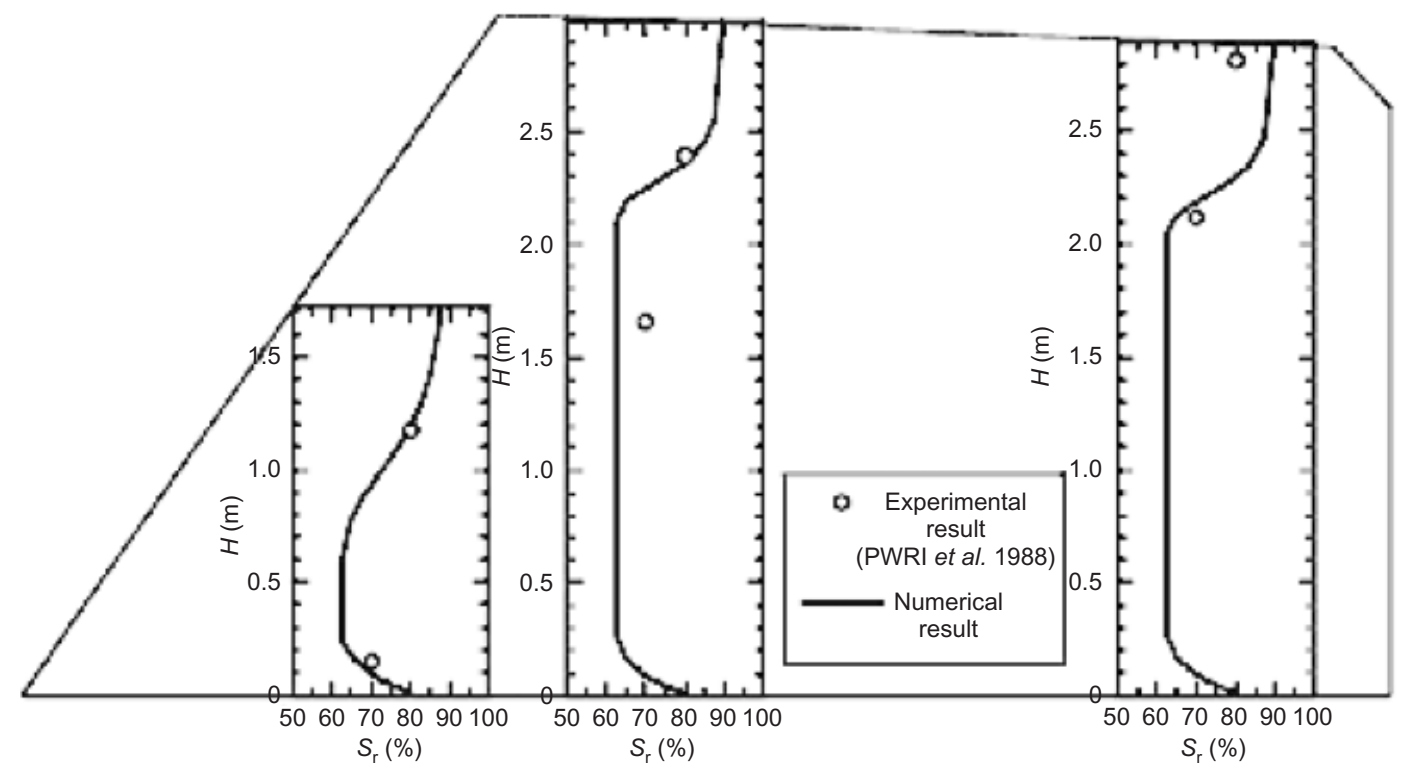

(a)

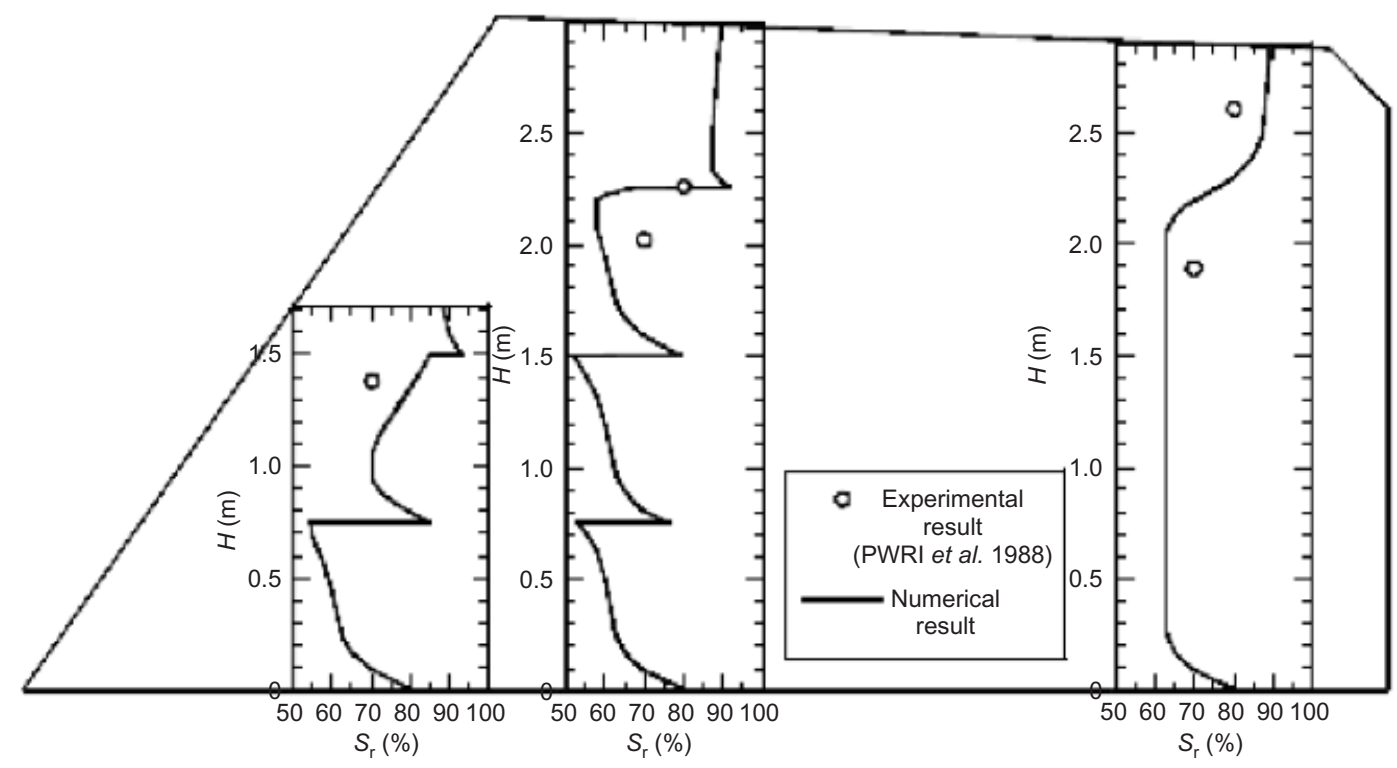

(b)

Figure 18. Comparison between water profiles in a retaining wall: (a) without reinforcements; (b) with geotextile reinforcements (Iryo and Rowe 2005b)

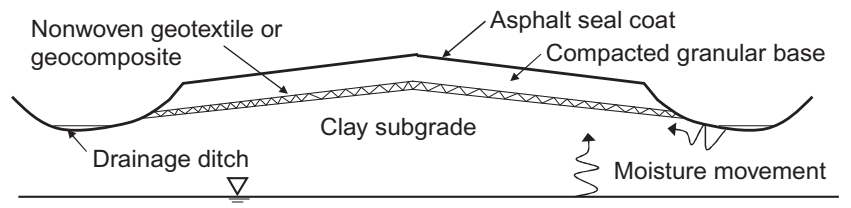

Figure 19. Nonwoven geotextile used as a hydraulic barrier in a flexible pavement

rise from underlying soils. They found that upward flow was blocked by placing a geotextile a few centimeters above the water table, and above the level at which the water content approaches saturation. McCartney et al. (2008) evaluated the use of nonwoven geotextiles and geocomposite drainage layers as water barriers in geotechnical applications where capillary flow is expected. This study used a similar approach to that of Henry and Holtz (2001) to observe the impact of capillary rise on layered soil-geosynthetic systems and soil-only control systems. Capillary rise was observed to occur rapidly in a control model, whereas capillary rise occurred more slowly when a nonwoven geotextile was placed between two soil layers (Figure 20). This was attributed to the low hydraulic conductivity of the geotextile under unsaturated conditions. Consistent with observations by Henry and Holtz (2001), capillary rise was prevented when using a geocomposite between two soil layers, owing to the air gap in the geonet. Filter paper measurements indicated that negative water pressure was transmitted from the soil 




Figure 20. Water content changes due to capillary rise in different soil-geosynthetic systems (McCartney et al. 2008)

through the nonwoven geotextile, but not across the geocomposite.

In addition to causing a capillary break, geosynthetics have been used in pavements to drain water from unsaturated soils by wicking action (Zerfass 1986). In this case, the polymer type must be carefully selected to ensure that the geosynthetic is wettable. Stormont et al. (2007) used geocomposites with fiberglass geotextiles to induce a wicking action that would drain water from unsaturated, compacted base course layers in a pavement system. Fiberglass has a higher affinity to water than other geosynthetic polymers (Henry and Patton 1998; Stormont and Ramos 2004). The geocomposite capillary barrier drain removes water from soil while pore pressures remain negative, leading to higher stiffness in the system. The geocomposite system comprises a capillary barrier layer (a geonet) sandwiched between transport layers (certain geotextiles). This study involved an experimental component in which water was infiltrated on the top of a pavement base course, and drainage from the geocomposite and the soil layers was collected. The geocomposite was successful in draining sufficient water under suction to prevent development of positive pore water pressures in the base course, and to limit water movement into the underlying subgrade soil.

\subsubsection{Impact on landfill leak-detection systems}

Rowe and Iryo (2005) noted that a geosynthetic capillary barrier may lead to unexpected behavior in the leakdetection or secondary leachate collection system below a landfill composite liner. Finite element simulations were used to conduct a parametric study that included assessment of the impact of the initial conditions in the underlying foundation soils and of the distance from the leakage point to the drainage point on the time for leakage to be detected. They found that the time for leakage depends on the initial degree of saturation of the material. A good comparison was obtained between predicted leakage using numerical simulations and field monitoring results. It was concluded that the time at which leakage occurs from primary landfill liner systems may be seriously overestimated.

\subsubsection{Impact on agriculture and turf underdrain} applications

McCartney and Allen (2008) evaluated the use of geosynthetic capillary barriers to increase water storage within the plant rooting zone. Column tests were performed using low-plasticity soil obtained from a test farm to evaluate the impact of the thickness of the soil layer atop a geosynthetic layer on plant growth. The geosynthetic capillary barrier was observed to cause an increase in soilwater storage to an elevation of nearly $0.5 \mathrm{~m}$ above the geosynthetic. Also, the response of a vegetated soil column containing a geosynthetic capillary break was compared with another column without a capillary break. The irrigation volumes, surface radiation (from growing lamps), and water storage were tracked from the time of planting over a three-month time period. The results indicated that plants grew more healthily in the columns with a geosynthetic capillary break. The results from this study can be extended to the design of turf systems for sports fields. Selection of the depth of the geosynthetic layer is a key design element to achieve adequate performance of turf underdrain systems.

\subsection{Impact of geosynthetic capillary barriers on the design of alternative covers}

Two important aspects related to geosynthetic capillary barriers significantly affect the assessment of alternative covers involving evapotranspirative systems. One of these aspects is the fact that lysimeters, the monitoring instruments that have been used to demonstrate the acceptable performance of these covers, have been providing erroneously low (unconservative) percolation values as a result of the development of an often-overlooked capillary barrier. The second aspect concerns the superior performance of geosynthetic capillary barriers in relation to soilonly capillary barriers. These two aspects are discussed next.

\subsubsection{Impact of geosynthetic capillary barriers on} lysimetry

A discussion of lysimetry is necessary within the context of this paper, as this is a monitoring device that, at least in the US and more recently in Australia, has provided the basis for most of the equivalence demonstrations that led to the acceptance of alternative cover systems. US regulations for municipal and hazardous waste landfills allow the use of alternative covers as long as it is demonstrated that the alternative 'achieves an equivalent reduction in infiltration' as the prescriptive cover. However, this equivalence demonstration has recently become a source of controversy, and at the centre of this controversy is the capillary barrier effect induced by nonwoven geotextiles. Accordingly, understanding of the capillary barrier phenomenon may have profound implications for current landfill design practice. This is because lysimetry is the currently accepted field monitoring method for equivalence demonstration of an alternative cover. Specifically, the demonstration often involves the construction of a suite of proposed alternative covers and subsequent mon- 
itoring, with lysimetry, of the basal percolation under representative weather conditions.

In a nutshell, a lysimeter is a device placed under a soil layer that collects water that has percolated through the layer. Lysimeters were first used in agronomy, although engineers began using them to prove equivalence of alternative earthen final covers, such as the monolithic covers described above in Section 4.1. There are various different types of lysimeter (e.g. pan lysimeters, suction lysimeters), but pan lysimeters have been the most commonly used type to measure percolation through alternative covers. The pan lysimeter consists of a geocomposite drainage layer that is overlain by the proposed cover and is underlain by a relatively impermeable layer, usually a geomembrane (Gee and Hillel 1988). The expectation has been that percolating flow, moving downwards through the proposed monolithic cover, would be collected by the lysimeter. The collected flow would then be directed into a collection tank, where the volume of collected water would be quantified. The schematic view of a pan lysimeter is shown in Figure 21 (Benson et al. 2001). As seen in the figure, the lysimeter is placed at a minimum inclination so that the water collected in the geocomposite drain can be carried by gravity to the percolation pipe and into the collection basin. In this particular example the lysimeter includes a low linear density polyethylene (LLDPE) geomembrane as the impervious layer, both under the geocomposite drainage layer and in the sidewalls located at the perimeter of the lysimeter. Sidewalls are used to prevent lateral diversion of water. Lysimeters became popular because they provide a direct measurement of the variable of interest (i.e. basal percolation through the proposed cover). Also, lysimeters can be constructed with a size that is large enough to account for spatial variability of the soil layer. Despite these potential advantages, there are several major drawbacks that may have been overlooked in the current state of the practice.

Lysimeters have been proven adequate for the comparatively high flow rates induced in irrigation for agronomy studies. They were then used for evaluation of alternative cover systems, because they were thought to be an adequate approach for direct measurement of basal percolation through a soil profile (Gee and Hillel 1988). In 1998 the EPA-funded Alternative Cover Assessment Program (ACAP) initiated research into the effectiveness of certain alternative covers. The goal of ACAP was to produce field data from field-scale tests of alternative and prescriptive covers. Over 19 sites, ranging from municipal solid waste landfills to hazardous waste landfills, were instrumented using lysimetry. In some cases, sensors were also used for measurement of water content and suction. ACAP focused on ET covers, and included a variety of covers with varying dimensions. Many of the sites involved monolithic covers, although some projects involved capillary barrier covers of varying depths. For the monitoring of percolation, ACAP requires that each site contain at least one test section $(10 \mathrm{~m} \times 20 \mathrm{~m})$ that consists of a large-scale, pan-type lysimeter. It appears that little attention has been given in this program to how the lysimeters themselves may affect the flow of water in the covers. However, recent infiltration studies, such as those discussed in Section 5 of this paper, have brought to light concerns regarding the adequacy of relying heavily on lysimetry for evaluation and acceptance of alternative covers. As discussed in the column studies reported in Section 5, a capillary barrier develops at the interface between the cover soil and the underlying geocomposite drainage layer. In addition to laboratory infiltration studies, data collected from lysimeters and water profiles installed at the high-visibility Rocky Mountain Arsenal site (Zornberg and McCartney 2003) reveal that the water content at the field lysimeters followed the same pattern as that shown in Figure 16. Specifically, water had accumulated at the base of the cover (i.e. the top of the lysimeter), owing to the development of a capillary barrier on the very instrument meant to monitor percolation. In other words, this compromised measurement of the actual percolation that would have occurred through the base of the cover soil had the lysimeter not been installed (i.e.

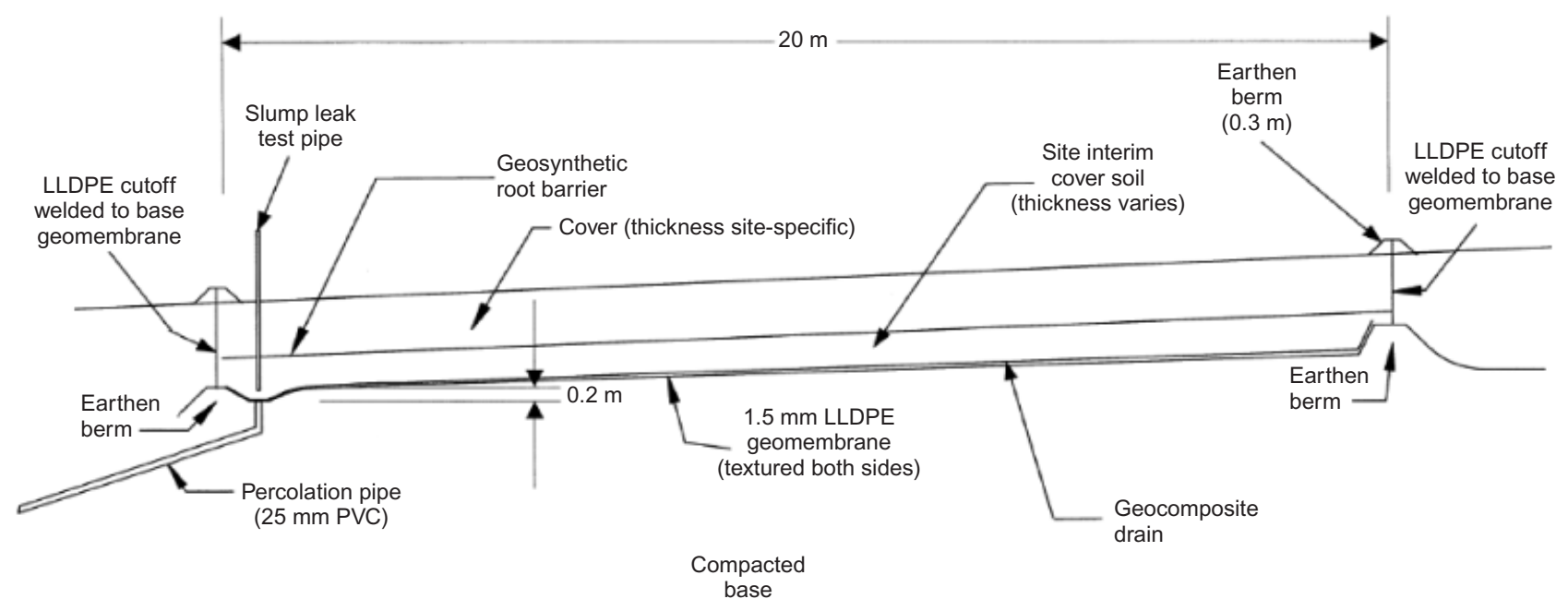

Figure 21. Schematic view of a typical pan lysimeter used for evaluation of the performance of alternative cover systems (Benson et al. 2001) 
field conditions representative of a monolithic cover system). Consequently, the development of a capillary barrier has led to an underestimation of the actual basal percolation through the cover. Accordingly, an alternative cover that has been proven to be acceptable (i.e. demonstrated equivalence) using only lysimeter measurements may indeed have an unacceptably high percolation in the field, where the beneficial effect of the capillary break will not develop.

By their nature, lysimeters are intrusive measurement devices, since the flow pattern within a soil layer located over a lysimeter will differ from that within the same soil layer without a lysimeter. Because of the development of a capillary barrier, the authors recommend that lysimetry be used with caution in test plots involving proposed monolithic covers, as these covers will not have an engineered capillary barrier. Also, the use of lysimetry should be carefully evaluated even in test plots for covers including a capillary barrier, as the nature of the capillary barrier that will develop over the lysimeter should be proven to be equivalent to the one that is expected to develop in the constructed cover. Indeed, as will be discussed in Section 6.2.2, geosynthetic capillary barriers have been shown to provide higher water storage than soil-only capillary barriers. Construction of duplicate test covers, both with water content sensors but only one of them with an underlying lysimeter, may prove useful for proper evaluation of the proposed alternative covers. In summary, while the use of geosynthetic capillary barriers is beneficial, as it leads to increased water storage in alternative covers, the use of lysimetry in current practice may have led to alternative covers that are unconservatively designed.

\subsubsection{Impact of geosynthetic capillary barriers on water storage}

As indicated by the results of column tests such as those shown in Figure 16, the development of a capillary barrier enhances the performance of an alternative cover system (e.g. a monolithic cover), since the water storage within the finer-grained material is increased in relation to that associated with free drainage. The increased storage capacity in the overlying material makes additional precipitation water available for subsequent release to the atmosphere as evapotranspiration, rather than for continued downward infiltration into the waste.

However, even in projects involving the construction of a capillary barrier, the amount of water storage for a given fine-grained soil will depend on the properties of the selected capillary barrier material. More specifically, as illustrated by Figure 3, the amount of water storage will depend on the WRC and $K$-function of the capillary barrier material. A study was conducted by McCartney et al. (2005) to compare the performance of geosynthetic capillary barriers with that of soil-only capillary barriers. Figure 22 shows a schematic view of two profiles that were constructed for this study. Column 1 includes finegrained low-plasticity clay placed over a sand layer acting as capillary barrier. Specifically, a $300 \mathrm{~mm}$ layer of clay was placed in $50 \mathrm{~mm}$ lifts over the sand layer, using static compaction, to the target dry unit weight of $75 \%$ of the



Figure 22. Schematic view of infiltration columns (McCartney et al. 2005)

maximum dry unit weight (based on the standard Proctor compaction effort) and a gravimetric water content of $8 \%$ (volumetric water content of $12 \%$ ). Column 2 includes the same fine-grained soil, but placed over a geocomposite drainage layer that rests on a gravel foundation layer. Volumetric water content values were continuously measured throughout the vertical soil profiles using TDRs. Figure 22 shows the location of the TDR probes in both columns. In Column 1 four TDR probes were used, including two probes located immediately above and below the interface between the clay and sand layers to monitor the interface behavior. In Column 2 three probes were used, including a probe located immediately above the geocomposite. A peristaltic pump was used to supply a relatively constant flow rate of $0.4 \mathrm{~cm}^{3} / \mathrm{s}$ to the top surface of the clay. This water supply corresponds to a Darcian velocity of $2.06 \times 10^{-7} \mathrm{~m} / \mathrm{s}$. The flow rate was selected to be less than the saturated hydraulic conductivity of the clay, to maintain unsaturated soil conditions. As reported by McCartney et al. (2005), the low-plasticity clay had a relatively low saturated hydraulic conductivity of $1.2 \times 10^{-6} \mathrm{~m} / \mathrm{s}$, whereas the sand had a saturated hydraulic conductivity of $5.3 \times 10^{-4} \mathrm{~m} / \mathrm{s}$. The geocomposite drainage layer used in this study involved a geonet sandwiched between two nonwoven geotextiles with a cross-plane hydraulic conductivity of $1.9 \times 10^{-3} \mathrm{~m} / \mathrm{s}$.

Although this study involves infiltration into dry soil (i.e. following the wetting path of the soil WRC), the drying-path WRC was deemed appropriate to highlight important hydraulic differences between the two different materials used as capillary barriers. Figure 3 showed the water retention data of the three geomaterials used in this study, along with the best-fit WRCs defined using the van Genuchten (1980) model. Also, the results previously shown in Figure 9 correspond to the $K$-functions of the three geomaterials used in this study. They were defined using the WRC parameters and the saturated hydraulic conductivity $\left(K_{\mathrm{s}}\right)$ values obtained from flexible wall permeameter tests for both the clay and the sand. The geotextile saturated hydraulic conductivity was based on the permittivity measurement as reported by the geocomposite manufacturer. As previously discussed, the results 
in Figure 9 indicate that, as suction increases, the hydraulic conductivity values of the three materials decrease at different rates.

The $K$-functions in Figure 9 indicate that a capillary break is likely at the interface between the clay and the nonwoven geotextile, as well as between the clay and the sand. While suction across the interface of two geomaterials in contact is the same, Figure 9 highlights that the three tested materials may have different hydraulic conductivities for a given value of suction, except when their curves intersect. Specifically, as a result of downward flow through the initially dry (high suction) clay layer, water will not flow into the underlying layer until the suction decreases to the value at which the conductivity of both layers is the same. This is the case for the interface between the clay and the sand, and for that between the clay and the geotextile component of the geosynthetic drainage layer. It should be noted in Figure 9 that the hydraulic conductivity of both the geotextile and the sand decrease sharply with increasing suction, although such drop occurs at lower suction values in the case of the geotextile than in the case of the sand.

Figure 23 shows the changes in water content with time at four different elevations in Column 1. This figure indicates that the sand is initially very dry (volumetric water content of approximately $5 \%$ ), which corresponds to a negligible hydraulic conductivity. The initial volumetric water content of the clay soil is approximately $12 \%$ throughout the entire thickness of the profile. The volumetric water content measured by TDR 1 (near the soil surface) increases to approximately $25 \%$ as the water front advances through the clay. Similarly, the volumetric water content measured by TDR 2 increases to $25 \%$ after a period of about $5000 \mathrm{~min}$. The volumetric water content measured by TDR 3 also increases to $25 \%$, but because of the proximity to the capillary barrier it shows a continued increase in water content to approximately $36 \%$. Subsequently, after approximately $7000 \mathrm{~min}$, TDR 2 shows increasing water content, consistent with TDR 3 readings. This behavior indicates that the wetting front reached the sand interface, but water accumulated above the interface rather than flowing directly into the sand layer. After the clay reached a volumetric water content of $36 \%$ near the

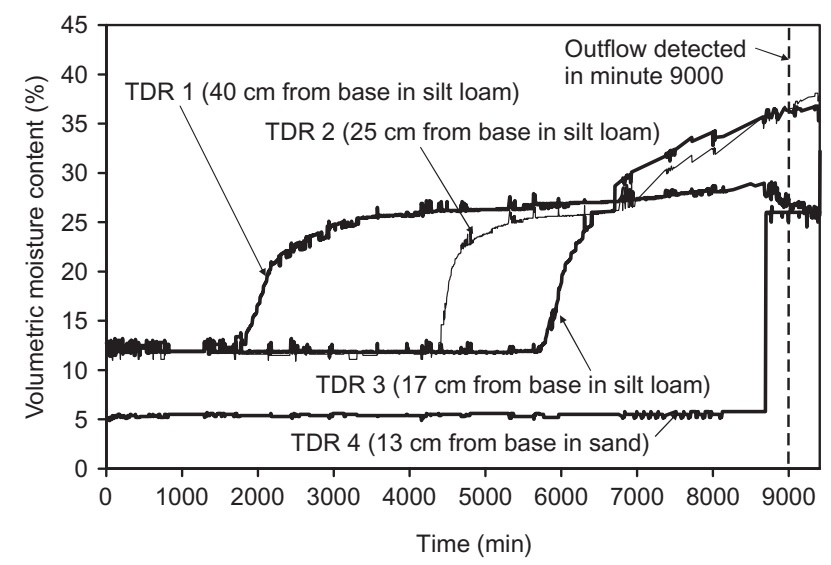

Figure 23. Volumetric water content with depth in Column 1 (McCartney et al. 2005) interface, breakthrough is noted by a sudden increase in the volumetric water content $(26 \%)$ recorded by TDR 4 located within the sand layer. The increase in volumetric water content within the sand layer occurred around the same time as outflow was collected at the base of the profile (after approximately $9000 \mathrm{~min}$ ). The performance of Column 1 is consistent with the development of a capillary break, and indicates that the clay layer has a volumetric water content of approximately $36 \%$ at breakthrough. The WRC of the clay (Figure 3) indicates that this volumetric water content corresponds to a suction of approximately $5 \mathrm{kPa}$. In turn, this suction value is consistent with the suction value at which the $K$-functions of the clay and sand intersect (Figure 9).

Figure 24 shows the changes in water content with time at three different elevations in Column 2. Also in this case the initial volumetric water content of the clay soil is approximately $12 \%$. Consistent with the response shown by Column 1, the volumetric water content recorded in Column 2 by TDR 5 (near the soil surface) increases to approximately $25 \%$ as the water front advances through the clay. Similarly, the volumetric water content measured by TDR 2 increases to $25 \%$ after a period of about $3500 \mathrm{~min}$. Finally, the volumetric water content measured by TDR 7 (near the geocomposite) also increases, but its continued increase is to a water content that is higher than reached near the interface of Column 1 (around 40\%). Also, unlike the response shown in Column 1, not only TDR 6 but also TDR 5 (near the soil surface) shows an increase in water content from $25 \%$ to $40 \%$. Consequently, essentially the entire Column 2 was affected by the capillary break induced by the geocomposite, indicating that use of a geocomposite (rather than sand) as capillary barrier led to increased water storage within the clay layer. Outflow from Column 2 was detected after 8180 min. As shown by the clay WRC (Figure 3), a water content of approximately $40 \%$ corresponds to a suction value of about $3 \mathrm{kPa}$. This suction value is slightly lower than that obtained at breakthrough in Column 1, and is consistent with the intersection of the $K$-functions for the clay and the geotextile (Figure 9).

Figure 25 shows the water storage within the clay soil as a function of time for both columns, calculated by

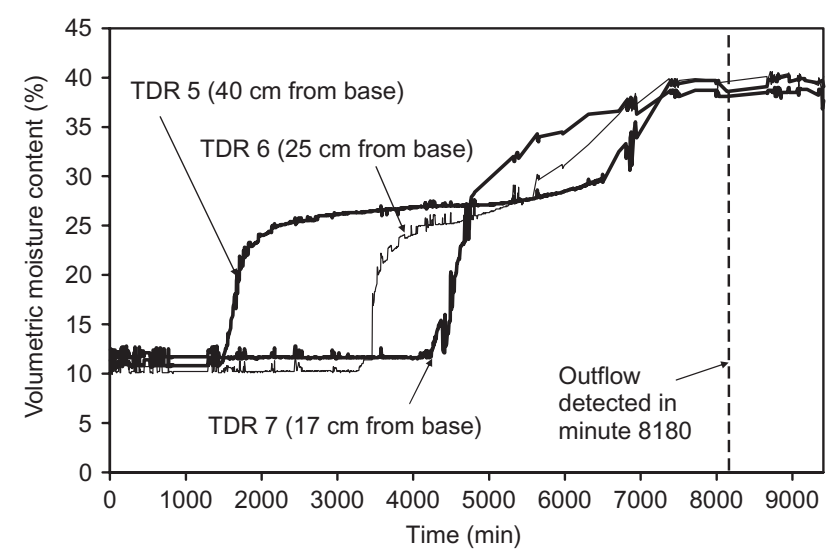

Figure 24. Volumetric water content with depth in Column 2 (McCartney et al. 2005) 


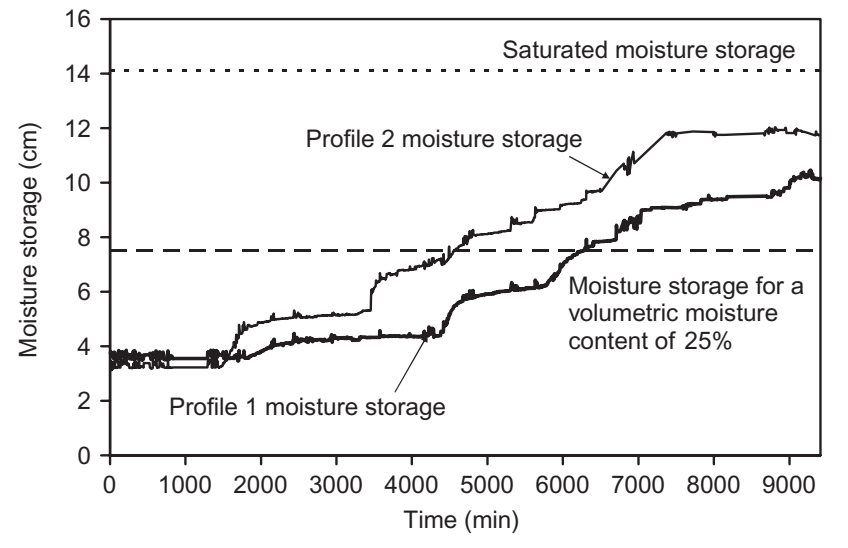

Figure 25. Water storage in Columns 1 and 2 during infiltration and at saturation (McCartney et al. 2005)

integrating the water content profile with depth. This figure shows that the water storage increases as the infiltration front advances through the soil. Two values of water storage are shown as reference in the figure: the storage corresponding to a water content of $25 \%$ (the water content associated with free draining of the imposed impinging flow rate), and the water storage corresponding to saturated conditions. The shape of the water storage curves for both profiles indicates that the clay stores water well in excess of the value expected from a freely draining condition. Also, as clearly shown by the water measurements in this study, the geosynthetic capillary barrier outperformed the sand capillary barrier. Similar results were obtained in an infiltration study reported by Krisdani et al. (2006).

In summary, geotextile capillary barriers provide higher water storage than that provided by granular soils. In addition, they also offer separation and filtration benefits that are necessary for good long-term performance of capillary barriers involving granular soils. Based on these findings, it is recommended that the inclusion of a nonwoven geotextiles at the base of the soil component of the cover is considered for all granular capillary barriers.

\subsubsection{The Rocky Mountain Arsenal case history}

The Rocky Mountain Arsenal (RMA) is a Superfund site located near Denver, Colorado, USA, which corresponds to one of the most highly contaminated hazardous waste sites in the US. One of the remediation components at the site involved the design and construction of alternative covers. This project is a good example of the previously discussed issues, because the use of lysimeters as part of the design phase brought to light the concerns associated with the impact of capillary break in the equivalence demonstration.

The project involved over 400 acres (160 ha) of RCRAequivalent evapotranspirative covers. The climate in Denver is semi-arid, with an average annual precipitation of $396 \mathrm{~mm}$ and an average pan evaporation of $1394 \mathrm{~mm}$ (as quantified for the 1948 to 1998 period). The wettest months of the year (April to October) are also the months with the highest pan evaporation, which is appropriate for an evapotranspirative cover. The Record of Decision
(ROD) for this hazardous waste site required a compliance demonstration to show equivalence of the alternative design with a prescriptive cover before construction of the final evapotranspirative covers. The design and compliance of the covers at the RMA site are governed by a quantitative percolation criterion. A threshold of $1.3 \mathrm{~mm} /$ year was selected at this site, which was based on 8 years of leachate data collected from two landfill covers built to RCRA Subtitle C standards in Hamburg, Germany, according to analyses described by Melchior (1997). This type of criterion was selected for its simplicity, as it sets a benchmark to be used in post-closure monitoring to demonstrate compliance, and is representative of the basal percolation for resistive covers.

The compliance demonstration at the RMA involved a field demonstration, which was complemented by comparative numerical analyses (Kiel et al. 2002). Four evapotranspirative test covers were constructed on a rolling plain at the site in the summer of 1998. A plan view of the four test covers, referred to as test covers A, B, C, and $\mathrm{D}$, is shown in Figure 26a. The covers were constructed using site-specific clays of low plasticity (CL), compacted atop large pan lysimeters $(9.1 \mathrm{~m} \times 15.2 \mathrm{~m})$ placed on a $3 \%$ grade to allow gravity drainage to a collection tank. Figure $26 \mathrm{~b}$ shows a schematic view of the monitoring layout used in the test covers. The instrumentation program involved monitoring of the basal percolation, precipitation, soil volumetric water content, and overland runoff in the four test covers. Basal percolation was collected in gravity lysimeters, which involved a geocomposite underlain by geomembrane. Rain and snow were monitored using an all-season rain gauge. Surface water was collected in polyethylene geomembrane swales constructed around the cover perimeters. Water content reflectometer (WCR) probes were used to measure volumetric water content profiles. Specifically, the covers were instrumented with nests of eight WCR probes. This included six WCR probes placed in a vertical profile and spaced evenly with depth. In addition, redundant WCR probes were placed at the same depth as the top and the bottom probes, approximately $0.3 \mathrm{~m}$ aside from the vertical profile of WCR probes. Cover D was instrumented using three vertical nests of WCR probes. Figure 26c shows an elevation view of the covers, indicating the depth of each cover. The covers are separated from each other by $2.4 \mathrm{~m}$-wide buffer zones, and the entire area is vegetated with local grasses and shrubs.

While the test plots were well instrumented, the equivalent demonstration process initially focused almost exclusively on the lysimeter measurements, since the goal was to verify that the water flux through site-specific soils under local weather conditions remains below the threshold of $1.3 \mathrm{~mm} /$ year (Kiel et al. 2002). According to the lysimeter measurements, all test plots at RMA satisfied the quantitative percolation criterion over the period 1998-2003 of operation. That is, all four lysimeters recorded a yearly basal percolation rate below the threshold, despite having complemented the natural precipitation with irrigation. Although Cover D showed surface depressions, possibly caused by installation of water probes, or 


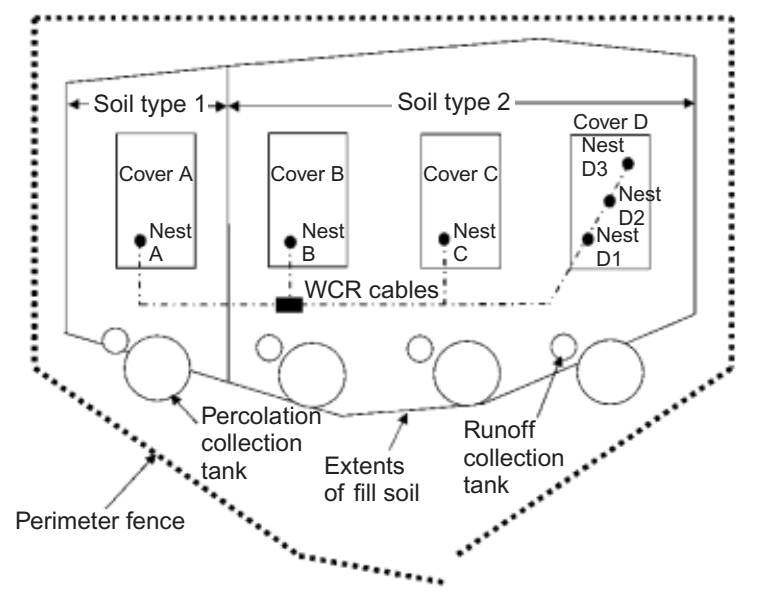

(a)

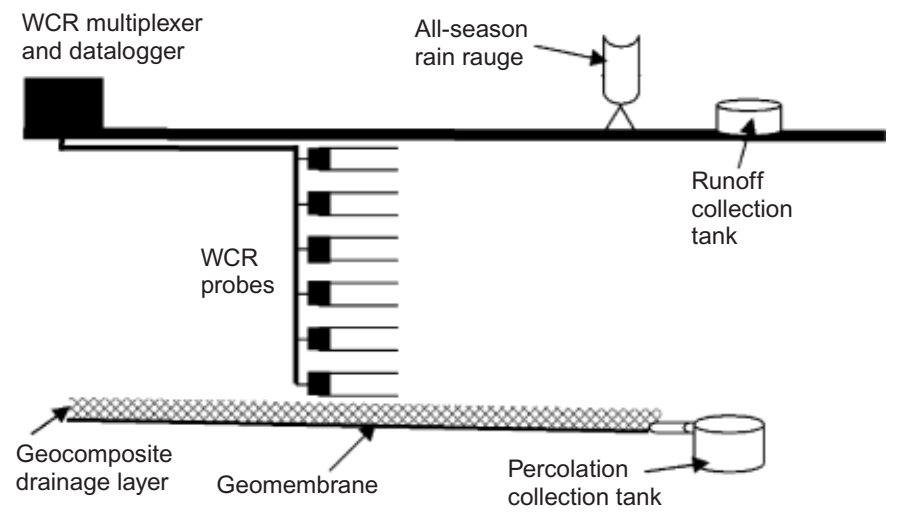

(b)

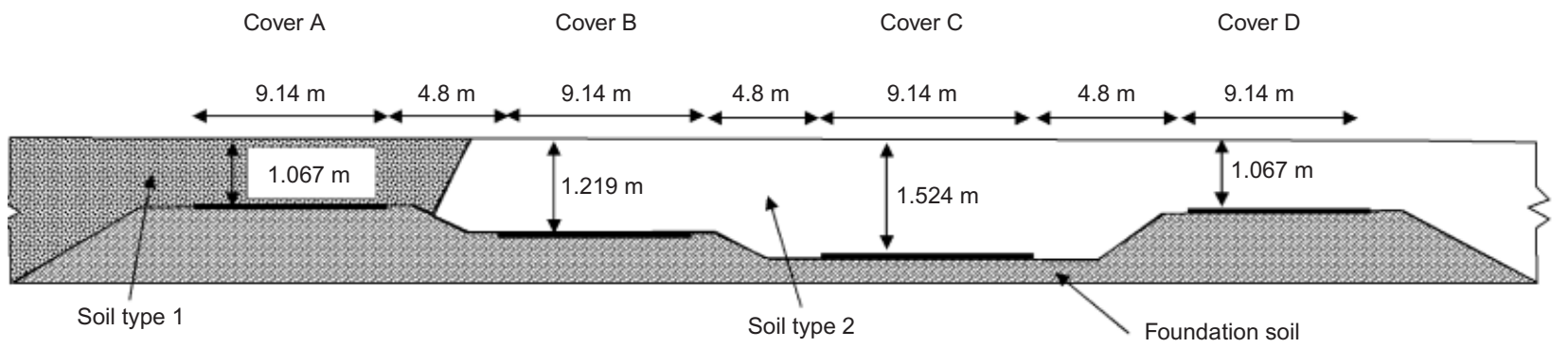

(c)

Figure 26. Layout for the monitoring program at the Rocky Mountain Arsenal: (a) plan view; (b) schematic view of instrumentation; (c) elevation view (Zornberg and McCartney 2003)

by burrowing animals, the collected basal percolation over this cover was still below the percolation threshold.

However, subsequent evaluation of the water content records revealed that the lysimeters had affected the natural flow of water by creating a capillary barrier (Zornberg and McCartney 2003). The water profiles (similar to those shown by the column study in Figure 16) led to underprediction of percolation for the alternative covers. Even though this effect was not identified until after the alternative covers had been approved, with no provision of an engineered capillary barrier, the cover design was amended to include a capillary barrier. In this way, the constructed cover system would have the same components as those of the tests plots used in the demonstration program, including the capillary break induced by the lysimeters.

The final cover design for the first group of alternative covers being constructed at the RMA is shown in Figure 27. As shown in the figure, the final cover design includes a geosynthetic capillary barrier. Specifically, the cover profile includes a $1.067 \mathrm{~m}$-thick barrier layer, similar to that used in covers A and D. An additional $0.152 \mathrm{~m}$-thick layer of topsoil is used for vegetation development. The specifications for the final cover soil are based on those for Soil Type 1 used in Test Cover A. The final design of the first cover constructed at the site includes a nonwoven geotextile over a chokestone layer (coarse gravel) to form a capillary break at the bottom interface of the barrier soil.
The geotextile also helps minimize the migration of soil particles into the chokestone layer. The chokestone is underlain by a biotic barrier consisting of crushed concrete from a demolition site. A biotic barrier was incorporated to prevent plants and burrowing animals from reaching the waste. The final cover is being instrumented using gravity lysimeters placed within the final cover to measure basal percolation, but also with WCR probes to measure water profiles within the barrier soil.

\section{CONCLUSIONS}

This paper provides theoretical background, laboratory data and full-scale measurements useful in understanding the interaction between soils and geosynthetics under unsaturated conditions. An evaluation is provided of the current state of knowledge regarding the hydraulic properties of porous geosynthetics under unsaturated conditions relevant for geosynthetic capillary barrier design. Specific applications are presented to illustrate new opportunities and applications that result from a better understanding of the unsaturated hydraulic properties of geosynthetics. The following conclusions can be drawn from this evaluation.

- The hydraulic conductivity of unsaturated geomaterials with relatively large pores (e.g. gravel, geotextiles) decreases faster than that of fine-grained soils. This phenomenon leads to the counterintuitive 


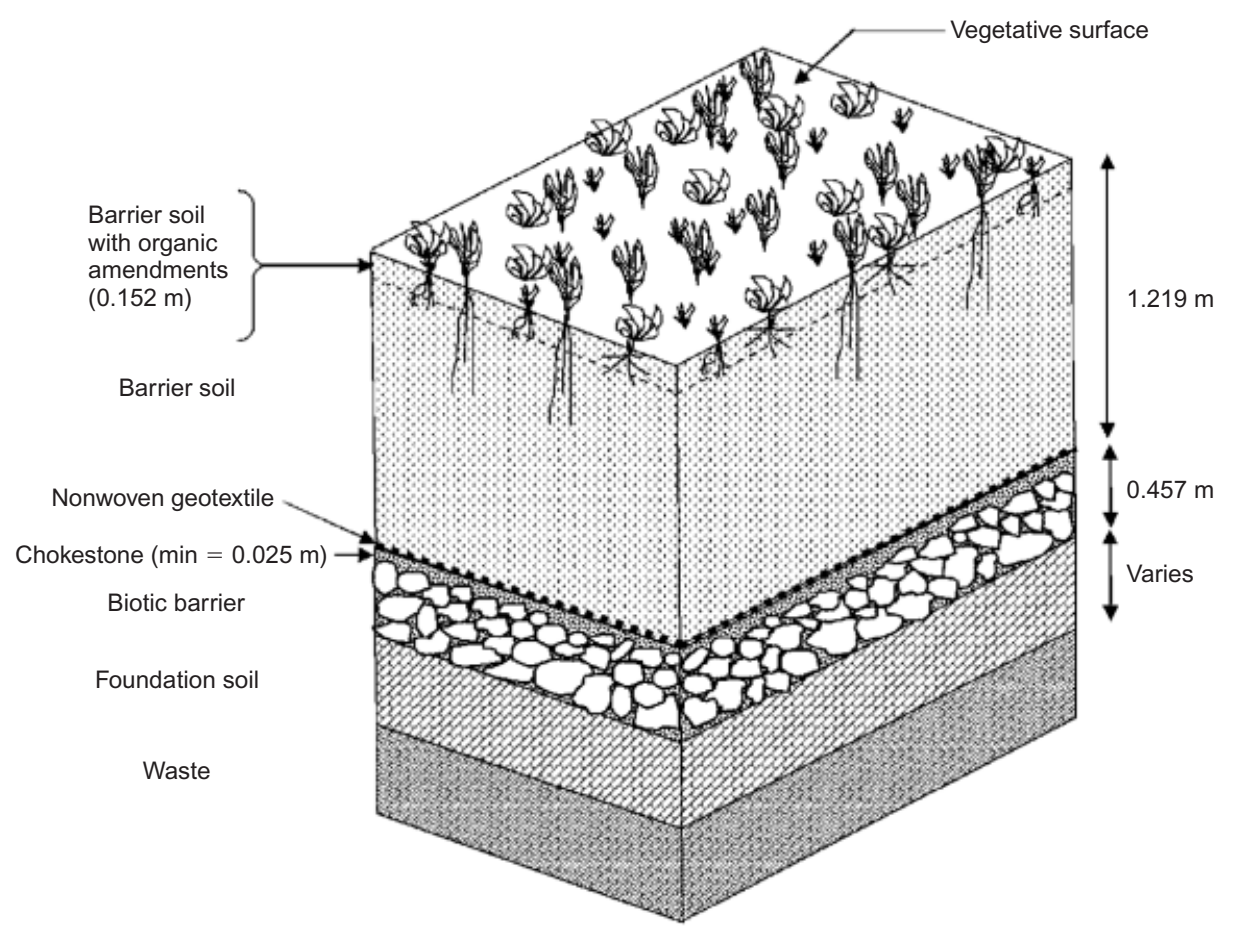

Figure 27. Final cover design at the Rocky Mountain Arsenal in Denver, Colorado, USA (Zornberg and McCartney 2003)

situation in which the hydraulic conductivity of unsaturated geotextiles can be significantly smaller than that of fine-grained soils.

- Several techniques have been developed to determine experimentally the WRCs of geotextiles, which are generally based on techniques originally developed for soils. These include hanging column tests, pressure plate tests, and capillary rise tests.

- As for soils, the $K$-function is still rarely obtained experimentally. Instead, the $K$-function of geotextiles has generally been defined using theoretical formulations based on the use of WRC parameters and the measured saturated hydraulic conductivity.

- The design and construction of alternative cover systems (e.g. monolithic covers, capillary barrier covers, anisotropic barrier covers) has grown significantly in arid and semi-arid regions. They account for evapotranspiration and water storage, two components of the water balance that are not formally considered in the design of resistive covers.

- Recent column studies have clearly shown the development of a capillary break at the interface between soils and an underlying nonwoven geotextile. Information from the WRC and $K$-function of the components of a capillary barrier can be used to predict the breakthrough suction and water storage expected in the fine-grained component.

- The development of geosynthetic capillary barriers may benefit a number of geotechnical and environmental applications. On the other hand, poor performance of earth structures involving nonwoven geotextiles may result from ignoring the capillary break effect. Geotechnical projects in which the development of capillary break is relevant include slopes with underdrains, reinforced-soil structures, pavements, landfill leak detection systems, and agricultural systems.

- The development of a capillary break in lysimeters used to monitor the performance of alternative covers has been often overlooked in current practice. This has led to erroneously low (unconservative) records of percolation in equivalence demonstrations.

- Results from infiltration studies demonstrate that geosynthetic capillary barriers in alternative covers typically outperform the soil-only capillary barriers.

The results from geotextile hydraulic characterization, column studies, and case histories, as presented in this paper, clearly document that capillary barriers develop at the interface between geotextiles and unsaturated soils. Consequently, proper design of capillary barrier covers should always consider the use of a nonwoven geotextile at the interface between fine-grained soils and the underlying coarse-grained capillary barrier material.

\section{NOTATION}

Basic SI units are given in parentheses.

$\begin{aligned} A & \text { area }\left(\mathrm{m}^{2}\right) \\ g & \text { acceleration of gravity }\left(\mathrm{m} / \mathrm{s}^{2}\right) \\ h_{\mathrm{c}} & \text { height of capillary rise }(\mathrm{m}) \\ K & \text { hydraulic conductivity }(\mathrm{m} / \mathrm{s}) \\ K_{\mathrm{s}} & \text { saturated hydraulic conductivity }(\mathrm{m} / \mathrm{s}) \\ K(\psi) & \text { hydraulic conductivity function }(K \text {-function }) \\ & (\mathrm{m} / \mathrm{s}) \\ N & \text { van Genuchten model parameter } \\ & (\text { dimensionless }) \\ n & \text { porosity (dimensionless) } \\ P_{\mathrm{a}} & \text { pore air pressure }(\mathrm{Pa})\end{aligned}$

$A$ area $\left(\mathrm{m}^{2}\right)$

$g$ acceleration of gravity $\left(\mathrm{m} / \mathrm{s}^{2}\right)$

$h_{\mathrm{c}}$ height of capillary rise $(\mathrm{m})$

$K$ hydraulic conductivity $(\mathrm{m} / \mathrm{s})$

$K_{\mathrm{s}} \quad$ saturated hydraulic conductivity $(\mathrm{m} / \mathrm{s})$

$K(\psi)$ hydraulic conductivity function ( $K$-function)

$(\mathrm{m} / \mathrm{s})$

van Genuchten

$n$ porosity (dimensionless)

$P_{\mathrm{a}}$ pore air pressure $(\mathrm{Pa})$ 
$P_{\mathrm{o}}$ osmotic suction $(\mathrm{Pa})$

$P_{\mathrm{w}}$ pore water pressure $\mathrm{kPa}$ )

$Q$ volumetric flow rate $\left(\mathrm{m}^{3} / \mathrm{s}\right)$

$R$ radius (m)

$S$ degree of saturation (dimensionless)

$v$ water discharge velocity $(\mathrm{m} / \mathrm{s})$

$z$ vertical distance from the datum (m)

$\alpha$ van Genuchten model parameter $\left(\mathrm{Pa}^{-1}\right)$

$\gamma \quad$ wetting contact angle (degrees)

$\theta$ volumetric water content (dimensionless)

$\theta_{\mathrm{r}}$ residual water content (dimensionless)

$\theta_{\mathrm{s}} \quad$ volumetric water content at saturation (dimensionless)

$\theta(\psi) \quad$ water retention curve (WRC) (dimensionless)

$\rho_{\mathrm{w}}$ density of water $\left(\mathrm{kg} / \mathrm{m}^{3}\right)$

$\sigma_{\text {aw }}$ surface tension between water and air $(\mathrm{N} / \mathrm{m})$

$\Phi$ fluid potential $\left(\mathrm{m}^{2} / \mathrm{s}^{2}\right)$

$\psi$ matric suction $(\mathrm{Pa})$

$\omega$ angular velocity $\left(\mathrm{s}^{-1}\right)$

\section{ACKNOWLEDGEMENTS}

This paper is a revised and updated version of the keynote paper presented during the First African Conference on Geosynthetics, GeoAfrica 2009, held in Cape Town, South Africa, in September 2009. The authors are grateful for the valuable input provided by the editor of the journal, R. J. Bathurst, and C. Pickles during preparation of this manuscript.

\section{REFERENCES}

Aydilek, A. H., D'Hondt, D. \& Holtz, R. D. (2007). Comparative evaluation of geotextile pore sizes using bubble point test and image analysis. Geotechnical Testing Journal, 30, No. 3, 173181.

ASTM D6836. Standard Test Methods for Determination of the Soil Water Characteristic Curve for Desorption Using a Hanging Column, Pressure Extractor, Chilled Mirror Hygrometer, and/or Centrifuge. ASTM International, West Conshohocken, PA, USA.

Anderson, J. E., Nowak, R. S., Ratzlaff, T. D. \& Markham, O. D. (1993). Managing soil water on waste burial sites in arid regions. Journal of Environmental Quality, 22, No. 1, 62-69.

Barbour, S. L. (1990). Reduction of acid generation in mine tailings through the use of moisture-retaining cover layers as oxygen barriers: Discussion. Canadian Geotechnical Journal, 27, No. 3, $398-401$.

Bathurst, R. J., Ho, A. F. \& Siemens, G. (2007). A column apparatus for investigation of 1-D unsaturated-saturated response of sandgeotextile systems. Geotechnical Testing Journal, 30, No. 6, 1-9.

Bathurst, R. J., Siemens, G. \& Ho, A. F. (2009). Experimental investigation of infiltration ponding in one-dimensional sandgeotextile columns. Geosynthetics International, 16, No. 3, 158172.

Benson, C. \& Gribb, M. (1997). Measuring unsaturated hydraulic conductivity in the laboratory and field. Unsaturated Soil Engineering Practice, Houston, S. and Wray, W., Editors, ASCE, Reston, VA, pp. 113-168.

Benson, C., Abichou, T., Albright, W., Gee, G. \& Roesler, A. (2001). Field evaluation of alternative earthen final covers. International Journal of Phytoremediation, 3, No. 1, 105-127.

Bouazza, A. (2004). Effect of wetting on gas transmissivity of non-woven geotextiles. Geotextiles and Geomembranes, 22, No. 6, 531-541.

Bouazza, A., Zornberg, J. G., McCartney, J. S. \& Nahlawi, H. (2006a).
Significance of unsaturated behaviour of geotextiles in earthen structures. Australian Geomechanics Journal, 41, No. 3, 133-142.

Bouazza, A., Freund, M. \& Nahlawi, H. (2006b). Water retention of nonwoven polyester geotextiles. Polymer Testing, 25, No. 8, 10381043.

Brooks, R. H. \& Corey, A. T. (1964). Hydraulic properties of porous medium. Colorado State University (Fort Collins). Hydrology Paper No. 3, March.

Burdine, N. T. (1953). Relative permeability calculations from pore-size distribution data. Petroleum Transactions of the American Institute of Mining and Metallurgical Engineering, 198, 71-77.

Bussiere, B., Aubertin, M. \& Chapuis, R. (2003). The behavior of inclined covers used as oxygen barriers. Canadian Geotechnical Journal, 40, No. 3, 512-535.

Cabral, A. R., Planchet, L, Marinho, F. A. \& Lefebvre, G. (2004). Determination of the soil water characteristic curve of compressible materials: case study of de-inking residues. ASTM Geotechnical Testing Journal, 27, No. 2, 154-162.

Choo, L. P. \& Yanful, E. K. (2000). Water flow through cover soils using modelling and experimental methods. Journal of Geotechnical and Geoenvironmental Engineering, ASCE, 126, No. 4, 324-334.

Christopher, B. R., Hayden, S. A. \& Zhao, A. (2000). Roadway and subgrade geocomposite drainage layers. Testing and Performance of Geosynthetics in Subsurface Drainage, ASTM STP 1390, L. D. Suits, J. B. Goddard and J. S. Baldwin, Editors, ASTM International, West Conshohocken, PA, USA.

Clough, I. R. \& French, W. J. (1982). Laboratory and field work relating to the use of geotextiles in arid regions. Proceedings of the 2nd International Conference on Geotextiles, IFAI, Las Vegas, NV, August 1983, Vol. 2, pp. 447-452.

Conca, J. \& Wright, J. (1992). Diffusion and flow in gravel, soil, and whole rock. Applied Hydrogeology, 1, 5-24.

Dell'Avanzi, E., Zornberg, J. G. \& Cabral, A. R. (2004). Suction profiles and scale factors for unsaturated flow under increased gravitational field. Soils and Foundations, 44, No. 3, 79-89.

Dwyer, S. F. (1998). Alternative landfill covers pass the test. Civil Engineering, 68, No. 9, 50-52.

Dwyer, S. F. (2001). Finding a better cover. Civil Engineering, 71, No. 1, $59-63$.

Fredlund, D. G. \& Xing, A. (1994). Equations for the soil-water characteristic curve. Canadian Geotechnical Journal, 31, No. 4, $521-532$.

Garcia, E. F., Gallage, C. P. K. \& Uchimura, T. (2007). Function of permeable geosynthetics in unsaturated embankments subjected to rainfall infiltration. Geosynthetics International, 14, No. 2, 89-99.

Gardner, R. (1937). A method of measuring the capillary tension of soil moisture over a wide moisture range. Soil Science, 43, No. 4, 277284.

Gardner, W. (1956). Calculation of capillary conductivity from pressure plate outflow data. Soil Science Society of America Proceedings, 20, 317-320.

Gee, W. \& Hillel, D. (1988). Groundwater recharge in arid regions: review and critique of estimation methods. Hydrological Processes, 2, No. 3, 255-266.

GRI (2003). The questionable strategy of soil-only landfill covers. A White Paper from the Geosynthetic Research Institute. Geotechnical Fabrics Report, March 2003, Vol. 21, pp. 18-23.

Hassler, G. L. \& Brunner, E. (1945). Measurements of capillary pressure in small core samples. Transactons of the AIME, 160, 114-123.

Henry, K. (1996). Geotextiles to mitigate frost effects in soils: A critical review. Transportation Research Record, No. 1534, pp. 5-11.

Henry, K. \& Holtz, R. (2001). Geocomposite capillary barriers to reduce frost heave in soils. Canadian Geotechnical Journal, 28, No. 4, 678-694.

Henry, K. S. \& Patton, S. (1998). Measurements of the contact angle of water on geotextile fibers. Geotechnical Testing Journal, 21, No. 1, $11-17$.

Henry, K. \& Stormont, J. (1998). Geocomposite capillary barrier drain. US Patent 6152653..

Hillel, D. (1998). Environmental Soil Physics, Academic Press, San Diego, CA. 
Iryo, T. \& Rowe, R. K. (2005a). Hydraulic behaviour of soilgeocomposite layers in slopes. Geosynthetics International, 12, No. $3,145-155$.

Iryo, T. \& Rowe, R. K. (2005b). Infiltration into an embankment reinforced with nonwoven geotextiles. Canadian Geotechnical Journal, 42, No. 4, 1145-1159.

Kampf, M. \& Holfelder, T. (1999). Designing capillary barriers. Seventh International Waste Management and Landfill Symposium, CISA, Environmental Sanitary Engineering Centre, Sardinia, pp. 381-388.

Khire, M. V., Meerdink, J. S., Benson, C. H. \& Bosscher, P. J. (1995). Unsaturated hydraulic conductivity and water balance predictions for earthen landfill final covers. In Soil Suction Applications in Geotechnical Engineering Practice, GSP 48, W. K. Wray and S. L. Houston, Editors, ASCE, Reston, VA, USA, pp. 38-57.

Khire, M. V., Benson, C. H. \& Bosscher, P. J. (1999). Field data from a capillary barrier and model predictions with UNSAT-H. Journal of Geotechnical and Geoenvironmental Engineering, ASCE, 125, No. $6,518-527$.

Khire, M., Benson, C. \& Bosscher, P. (2000). Capillary barriers in semiarid and arid climates: design variables and the water balance. Journal of Geotechnical and Geoenvironmental Engineering, ASCE, 126, No. 8, 695-708.

Kisch, M. (1959). The theory of seepage from clay-blanketed reservoirs. Géotechnique, 9, No. 1, 9-21.

Kiel, R. E., Chadwick, D. G., Lowrey, J., Mackey, C. V. \& Greer, L. M. (2002). Design of evapotranspirative (ET) covers at the Rocky Mountain Arsenal. Proceedings: SWANA 6th Annual Landfill Symposium (CD-ROM).

Klute, A. (1986). Water retention: laboratory methods. Methods of Soil Analysis, Part 1: Physical and Mineralogical Methods, SSSA, Madison, WI, pp. 635-662.

Knight, M. \& Kotha, S. (2001). Measurement of geotextile-water characteristic curves using a controlled outflow capillary pressure cell. Geosynthetics International, 8, No. 3, 271-282.

Koerner, R. (2005). Designing with geosynthetics, 5th edition, Prentice Hall, Upper Saddle River, NJ.

Kool, J. B. \& Parker, J. C. (1987). Development and evaluation of closedform expression for hysteretic soil hydraulic properties. Water Resources Research, 23, No. 1, 105-114.

Krisdani, H., Rahardjo, H. \& Leong, E.-C. (2006). Experimental study of 1-D capillary barrier model using geosynthetic material as the coarse-grained layer. Proceedings Unsaturated Soils 2006, Carefree, AZ, 1-3 April 2006, pp. 1683-1694.

Lafleur, J., Lebeau, M., Faure, Y. H., Savard, Y. \& Kehila, Y. (2000). Influence of matric suction on the drainage performance of polyester geotextiles. Proceedings 53rd Annual Conference of Canadian Geotechnical Society, Montreal, Canada, 15-18 October 2000, Vol. 2, pp. 1115-1122.

Lu, N. \& Likos, W. J. (2005). Unsaturated Soil Mechanics, Wiley, New York.

McCartney, J. S. \& Allen, J. M. (2008). Use of geosynthetic capillary barriers for increasing water storage capacity of soils in agriculture applications. GRI 21 Conference, Cancun, Mexico, 5 March 2008 (CD-ROM).

McCartney, J. S. \& Zornberg, J. G. (2010a). Effect of infiltration and evaporation on geosynthetic capillary barrier performance. Canadian Geotechnical Journal, in press.

McCartney, J. S. \& Zornberg, J. G. (2010b). Centrifuge permeameter for unsaturated soils II: Results and analysis. Journal of Geotechnical and Geoenvironmental Engineering, 136, No. 8, 1064-1076.

McCartney, J. S., Kuhn, J. A. \& Zornberg, J. G. (2005). Geosynthetic drainage layers in contact with unsaturated soils. Proceedings of the 16th International Conference on Soil Mechanics and Geotechnical Engineering (ISSMGE), Osaka, Japan, 12-17 September, pp. 2301-2305.

McCartney, J. S., Villar, L. F. S. \& Zornberg, J. G. (2008). Nonwoven geotextiles as hydraulic barriers to capillary rise. Proceedings of GeoAmericas 2008, the First PanAmerican Geosynthetics Conference and Exhibition, Cancún, Mexico, 2-5 March 2008, pp. $252-261$.

McCartney, J. S., Zornberg, J. G. \& Villar, L. (2007). Estimation of the hydraulic conductivity function of unsaturated clays using an infiltration column test. Proceedings of the 4th Brazilian Conference on Unsaturated Soils (NSAT), UFBA, Salvador, Brazil, 10.

Meerdink, J. S., Benson, C. H. \& Khire, M. V. (1996). Unsaturated hydraulic conductivity of two compacted barrier soils. Journal of Geotechnical and Geoenvironmental Engineering, ASCE, 122, No. 7, 565-576.

Melchior, S. (1997). In-situ studies of the performance of landfill caps (compacted clay liners, geomembranes, geosynthetic clay liner, capillary barriers). Land Contamination and Reclamation, 5, No. 3, 209-216.

Meyer, P. D. \& Gee, G. W. (1999). Flux-based estimation of field capacity. Journal of Geotechnical and Geoenvironmental Engineering, 125, No. 7, 595-599.

Mitchell, J. K. \& Zornberg, J. G. (1995). Reinforced soil structures with poorly draining backfills. Part II: Case histories and applications. Geosynthetics International, 2, No. 1, 265-307.

Moore, R. (1939). Water conduction from shallow water tables. Hilgardia, 12, 383-426.

Morris, C. (2000). Unsaturated flow in nonwoven geotextiles. GeoEng 2000, Melbourne, Australia (CD-ROM).

Nachabe, M. H. (1998). Refining the definition of field capacity in the literature. Journal of Irrigation and Drainage Engineering, 124, No. 4, 230-232.

Nahlawi, H., Bouazza, A. \& Kodikara, J. K. (2007a). Characterisation of geotextiles water retention using a modified capillary pressure cell. Geotextiles and Geomembranes, 25, No. 3, 186-193.

Nahlawi, H., Bouazza, A. \& Kodikara, J. K. (2007b). Surface water infiltration in a 1-dimensional soil-geotextile column. Proceedings of the 10th Australia New Zealand Conference on Geomechanics, Brisbane, Australia, 21-24 October 2007, pp. 368-373.

Nahlawi, H., Bouazza, A. \& Kodikara, J. K. (2008). Capillary rise in unsaturated nonwoven geotextiles. GeoAmericas 2008, Cancun, Mexico, pp. 281-289.

Nahlawi, H. (2009). Numerical and experimental investigation of the unsaturated hydraulic behaviour of geotextiles. $\mathrm{PhD}$ thesis. Department of Civil Engineering, Monash University, Australia.

Nicholson, R. V., Gillham, R. W., Cherry, J. A. \& Reardon, E. J. (1989). Reduction of acid generation in mine tailings through the use of moisture-retaining cover layers as oxygen barriers. Canadian Geotechnical Journal, 26, No. 1, 1-8.

Nimmo, J. R., Rubin, J. \& Hammermeister, D. P. (1987). Unsaturated flow in a centrifugal field: measurement of hydraulic conductivity and testing of Darcy's law. Water Resources Research, 23, No. 1, $124-134$.

Olson, R. E. \& Langfelder, L. J. (1965). Pore water pressures in unsaturated soils. Journal of the Soil Mechanics and Foundations Division, ASCE, 91, SM4, 127-151.

Palmeira, E. \& Gardoni, M. (2002). Drainage and filtration properties of non-woven geotextiles under confinement using different experimental techniques. Geotextiles and Geomembranes, 20, No. 2, 97115.

Parent, S. E. \& Cabral, A. (2005). Material selection for the design of inclined covers with capillary barrier effect. Waste Containment and Remediation (GSP 142), Proceedings of GeoFrontiers 2005, 24-26 January 2005, Austin, TX, ASCE, Reston, VA (CD-ROM).

Rasmusson, A. \& Eriksson, J.-C. (1987). Capillary barriers in covers for mine tailing dumps, National Swedish Environmental Protection Board Report 3307, Royal Institute of Technology, Stockholm, Sweden.

Richardson, G. N. (1997). Fundamental mistakes in slope design. Geotechnical Fabrics Report, 15, No. 2, 15-17.

Rowe, K. R \& Iryo, T. (2005). Effects of geosynthetics on the hydraulic performance of leak detection systems. Canadian Geotechnical Journal, 42, No. 5, 1359-1376.

Shackelford, C. D., Chang, C.-K. \& Chiu, T.-F. (1994). The capillary barrier effect in unsaturated flow through soil barriers. 1st ICEG Conference, Edmonton, CA, pp. 789-793.

Siemens, G. \& Bathurst, R. J. (2010). Numerical parametric investigation 
of infiltration in one-dimensional sand-geotextile columns. Geotextiles and Geomembranes, 28, No. 5, 420-474.

Stormont, J. (1995). The effect of constant anisotropy on capillary barrier performance. Water Resources Research, 32, No. 3, 783-785.

Stormont, J. C. \& Anderson, C. E. (1999). Capillary barrier effect from underlying coarser soil layer. Journal of Geotechnical and Geoenvironmental Engineering, 125, No. 8, 641-648.

Stormont, J. C. \& Morris, C. E. (1998). Method to estimate water storage capacity of capillary barriers. Journal of Geotechnical and Geoenvironmental Engineering, 124, No. 4, 297-302.

Stormont, J. C. \& Morris, C. E. (2000). Characterization of unsaturated nonwoven geotextiles. Advances in Unsaturated Geotechnics, Shackelford, C., Houston, S. and Chang, N., Editors, ASCE, pp. $153-164$.

Stormont, J. C. \& Ramos, R. (2004). Characterization of a fiberglass geotextile for unsaturated in-plane water transport. ASTM Geotechnical Testing Journal, 27, No. 2, 1-6.

Stormont, J. C., Henry, K. \& Evans, T. (1997). Water retention functions of four nonwoven polypropylene geotextiles. Geosynthetics International, 4, No. 6, 661-672.

Stormont, J. C., Ramos, R. \& Henry, K. S. (2007). Geocomposite capillary barrier drain system with fiberglass transport layer. Transportation Research Record, No. 1772, pp. 131-136.

Stormont, J. C., Hines, J. S., Pease, R. E., Kelsey, J. A \& Dowd, D. (2008). The effectiveness of a geotextile as a capillary barrier. GeoAmericas 2008, Cancun, Mexico (CD-ROM).

Thiel, R. (1999). Design and testing of a NWNP geotextile gas pressure relief layer below a geomembrane cover to improve slope stability. Proceedings of Sardinia '99 7th International Landfill Symposium, October 1999, Vol. III, pp. 343-350.

Topp, G. C. \& Miller, E. E. (1966). Hysteretic water characteristics and hydraulic conductivities for glass-bead media. Soil Science Society of America Proceedings, 30, 156-162.

van Genuchten, M. (1980). A closed-form equation for predicting the hydraulic conductivity of unsaturated soils. Soil Science Society of America Proceedings, 44, 892-898.

Wang, X. \& Benson, C. H. (2004). Leak-free pressure plate extractor for the soil water characteristic curve. Geotechnical Testing Journal, 27, No. 2, 1-10.

Watson, K. K. (1996). An instantaneous profile method for determining the hydraulic conductivity of unsaturated porous materials. Water Resources Research, 2, No. 3, 709-715.

Waugh, W. J., Petersen, K. L., Link, S. O., Bjornstad, B. N. \& Gee, G. W. (1994). Natural analogs of the long term performance of engineered covers. In In Situ Remediation: Scientific Basis for Current and Future Technologies, Gee, G. W. \& Wing, N. R., Editors, Battelle Press, Richland, WA, pp. 379-409.
Woyshner, M. R. \& Yanful, E. K. (1995). Modelling and field measurements of water percolation through an experimental soil cover on mine tailings. Canadian Geotechnical Journal, 32, No. 4, 601-609.

Yanful, E. K. (1993). Oxygen diffusion through soil covers on sulphidic mine tailings. Journal of Geotechnical Engineering, 119, No. 6, $1207-28$.

Zerfass, K.-Ch. (1986). Syphoning effect of geotextiles. Proceedings 3rd International Conference on Geotextiles, Vienna, Austria, pp. $1197-1202$.

Znidarcic, D., Illangasekare, T. \& Manna, M. (1991). Laboratory testing and parameter estimation for two-phase flow problems. Proceedings of the Geotechnical Engineering Congress, Boulder, CO, McLean, F. G., Campbell, D. A. \& Harris, D. W., Editors, ASCE, New York, pp. $1078-1089$.

Zornberg, J. G. \& Christopher, B. R. (2007). Chapter 37: Geosynthetics. The Handbook of Groundwater Engineering, 2nd edition, Jacques W. Delleur, Editor, CRC Press, Taylor \& Francis Group, Boca Raton, FL.

Zornberg, J. G. \& McCartney, J. S. (2003). Analysis of Monitoring Data from the Evapotranspirative Test Covers at the Rocky Mountain Arsenal Geotechnical Research Report, US Environmental Protection Agency, Region 8, December, 227 p.

Zornberg, J. G. \& McCartney, J. S. (2007). Chapter 34: Evapotranspirative cover systems for waste containment. The Handbook of Groundwater Engineering, 2nd edition, Jacques W. Delleur, Editor, CRC Press, Taylor \& Francis Group, Boca Raton, FL.

Zornberg, J. G. \& McCartney, J. S. (2010). Centrifuge permeameter for unsaturated soils I: Theory and experimental philosophy. Journal of Geotechnical and Geoenvironmental Engineering., 136, No. 8, 1051-1063.

Zornberg, J. G. \& Mitchell, J. K. (1994). Reinforced soil structures with poorly draining backfills. Part I: Reinforcement interactions and functions. Geosynthetics International, 1, No. 2, 103-148.

Zornberg, J. G., Jernigan, B. L., Sanglerat, T. R. \& Cooley, B. H. (1999). Retention of free liquids in landfills undergoing vertical expansion. Journal of Geotechnical and Geoenvironmental Engineering, ASCE, 125, No. 7, 583-594.

Zornberg, J. G., LaFountain, L. \& Caldwell, J. A. (2003). Analysis and design of evapotranspirative cover for hazardous waste landfill. Journal of Geotechnical and Geoenvironmental Engineering, 129, No. 5, 427-438.

Zornberg, J. G., Bouazza, A. \& McCartney, J. S. (2009). Geosynthetic capillary barriers: Principles and applications. Invited keynote lecture. Proceedings of the First African Conference on Geosynthetics, GeoAfrica 2009, Cape Town, South Africa, 2-4 September (CD ROM).

The Editor welcomes discussion on all papers published in Geosynthetics International. Please email your contribution to discussion@geosynthetics-international.com by 15 April 2011. 